\title{
Crack Initiation in Brittle Materials
}

\author{
Antonin Chambolle, Alessandro Giacomini \& Marcello \\ PONSIGLIONE
}

Communicated by M. ORTIZ

\begin{abstract}
In this paper we study the crack initiation in a hyper-elastic body governed by a Griffith-type energy. We prove that, during a load process through a time-dependent boundary datum of the type $t \rightarrow \operatorname{tg}(x)$ and in the absence of strong singularities (e.g., this is the case of homogeneous isotropic materials) the crack initiation is brutal, that is, a big crack appears after a positive time $t_{i}>0$. Conversely, in the presence of a point $x$ of strong singularity, a crack will depart from $x$ at the initial time of loading and with zero velocity. We prove these facts for admissible cracks belonging to the large class of closed one-dimensional sets with a finite number of connected components. The main tool we employ to address the problem is a local minimality result for the functional

$$
\mathcal{E}(v, \Gamma):=\int_{\Omega} f(x, \nabla v) \mathrm{d} x+k \mathcal{H}^{1}(\Gamma)
$$

where $\Omega \subseteq \mathbb{R}^{2}, k>0$ and $f$ is a suitable Carathéodory function. We prove that if the uncracked configuration $u$ of $\Omega$ relative to a boundary displacement $\psi$ has at most uniformly weak singularities, then configurations $\left(u_{\Gamma}, \Gamma\right)$ with $\mathcal{H}^{1}(\Gamma)$ small enough are such that $\mathcal{E}(u, \emptyset)<\mathcal{E}\left(u_{\Gamma}, \Gamma\right)$.
\end{abstract}

\section{Introduction}

Griffith's criterion for crack propagation in hyper-elastic bodies asserts that, during a load process, a crack $\Gamma$ can grow only if the energy dissipated to enlarge the crack, which is basically assumed to be proportional to the area of the cracked surface, is balanced by the corresponding release of bulk energy. According to Griffith's theory, if $\Omega$ represents a two-dimensional hyper-elastic body, $\psi$ is a boundary datum and $\Gamma$ is a curve in $\Omega$ parameterized by arc length, then the crack 
$\Gamma\left(l_{0}\right)$ is in equilibrium if

$$
k\left(l_{0}\right):=\limsup _{l \rightarrow 0^{+}} \frac{W\left(u\left(l_{0}\right)\right)-W\left(u\left(l_{0}+l\right)\right)}{l} \leqq k,
$$

where $u\left(l_{0}\right)$ and $u\left(l_{0}+l\right)$ are the displacements associated to $\psi$ and to the cracks $\Gamma\left(l_{0}\right)$ and $\Gamma\left(l_{0}+l\right)$, respectively, $W$ is the bulk energy functional and $k$ is the toughness of the material. A quasistatic crack evolution is determined by an increasing function $t \rightarrow l(t)$ satisfying the Griffith's criterion for crack propagation, which asserts that for every $t$ we have $k(l(t)) \leqq k$ and

$$
(k-k(l(t))) \dot{l}(t)=0,
$$

that is, $\Gamma(l(t))$ propagates only if (1.1) holds with equality.

The aim of this paper is to discuss this criterion in the case of crack initiation, that is, when there is not a pre-existing crack in the body $\left(l_{0}=0\right)$. A fundamental role in the problem is played by the singularities of the body, namely the behavior of the elastic energy concentration of the deformation. Experiments show that small cracks usually appear near sufficiently strong singular points of the body, whose positions are essentially determined by its inhomogeneities. If the singularities of the body are sufficiently weak (for instance this is the case of homogeneous isotropic materials), a lot of the results given in the literature of materials science show that the derivative in (1.1) for $l_{0}=0$ is equal to zero. The conclusion is that Griffith's criterion is not adequate to predict crack initiation (and, as a consequence, a crack evolution originating from an uncracked configuration). These results require that the path of the crack is sufficiently regular (a line or a smooth curve). In this paper we prove that the same conclusion holds in the class of all one-dimensional closed sets with a finite number of connected components. More precisely we prove that the limit in (1.1) is zero if $\Gamma(l)$ is any family of closed sets with length less than $l$ and with at most $m$ connected components, with $m$ independent of $l$. In particular we do not prescribe the path nor the shape of the cracks.

Although it is more general, our study is in part motivated by the variational model for quasistatic crack propagation proposed by FranCFORT and MARIGO in [20]. The main features of this model are that the path of the increasing crack $\Gamma(t)$ is not preassigned, the class of admissible cracks is given by all sets with finite length, and the growth is not assumed to be progressive, namely the length of the crack is not assumed to be continuous in time. The classical Griffith's equilibrium condition for the configuration $(u(t), \Gamma(t))$ is replaced by a unilateral minimality property and an energy balance condition. The unilateral minimality property states that, during the crack evolution, the total energy is minimal among all configurations with larger cracks, namely

$$
W(u(t))+k \mathcal{H}^{1}(\Gamma(t)) \leqq W(v)+k \mathcal{H}^{1}(H),
$$

for every crack $H$ containing $\Gamma(t)$ and for every deformation $v$ admissible for the boundary datum $\psi$ and for $H$. (Here $\mathcal{H}^{1}$-the one-dimensional Hausdorff measure-is a suitable generalization of the length.) The energy balance condition states that the energy of the system evolves in relation with the power of external 
loads in such a way that no dissipation occurs (except for the surface energy spent to enlarge the crack). The authors claim that their model improves the understanding of the crack initiation with respect to the classical Griffith's criterion: in fact, in contrast with Griffith's model, it admits brutal crack initiation, that is, evolutions $\Gamma(t)$ of the type

$$
\Gamma(t)=\emptyset \quad \text { for every } t \leqq t_{i}
$$

and

$$
\inf _{t>t_{i}} \mathcal{H}^{1}(\Gamma(t))>0
$$

where $t_{i}$ is referred to as time initiation of the crack. In this paper we prove that, within the class of cracks which are closed and with at most $m$ connected components, crack initiation is always brutal whenever the elastic displacement presents sufficiently weak singularities. More precisely, we show that (1.2) can be violated only by cracks whose length is greater than a critical quantity $l^{*}$, depending on the boundary datum and on the physical properties of the material. On the contrary, in the presence of a point $x$ of strong singularity the crack initiation is progressive: a crack departs from $x$ at the initial time of loading and with zero velocity. These facts were proved by FrancForT and MARIGO in [20, Proposition 4.19], under the assumption that the path of the crack is given a priori by a finite number of fixed curves which can be parameterized by arc length. This is not the case in our larger class of admissible cracks.

The main tool we employ to address the problem of crack initiation is a local minimality result for the functional

$$
\int_{\Omega} f(x, \nabla v) \mathrm{d} x+k \mathcal{H}^{1}(\Gamma)
$$

where $\Omega$ is a bounded Lipschitz open set in $\mathbb{R}^{2}, k>0$, and $f: \Omega \times \mathbb{R}^{2} \rightarrow \mathbb{R}$ is a Carathéodory function strictly convex and $C^{1}$ in the second variable, satisfying standard $p$-growth estimates with $p>1$, and such that $f(x, 0)=0$. The functional (1.3) is a variant of a functional which first appeared in the theory of image segmentation, in a celebrated paper by MuMFord and SHAH [29]. The set $\Gamma$ belongs to the class

$$
\begin{aligned}
& \mathcal{K}_{m}(\bar{\Omega}):=\{\Gamma \subseteq \bar{\Omega}: \Gamma \text { has at most } m \text { connected components and } \\
& \left.\mathcal{H}^{1}(\Gamma)<+\infty\right\}
\end{aligned}
$$

and the function $v$ belongs to the Sobolev space $W^{1, p}(\Omega \backslash \Gamma)$ and satisfies the boundary condition

$$
v=\psi \quad \text { on } \partial_{D} \Omega \backslash \Gamma
$$

where $\partial_{D} \Omega \subseteq \partial \Omega$ is open in the relative topology, and $\psi$ is (the trace of a function) in $W^{1, p}(\Omega) \cap L^{\infty}(\Omega)$. 
Let $u_{\Gamma}$ be a minimum energy displacement relative to $\psi$ and $\Gamma$, that is, let $u_{\Gamma}$ be a minimizer for

$$
\min \left\{\int_{\Omega} f(x, \nabla v) \mathrm{d} x: u \in W^{1, p}(\Omega \backslash \Gamma), v=\psi \text { on } \partial_{D} \Omega\right\} .
$$

We denote by $u$ the elastic configuration of $\Omega$ relative to the boundary datum $\psi$, that is, a solution of (1.6) with $\Gamma=\emptyset$, and we assume that $u$ admits at most uniformly weak singularities in $\Omega$, that is,

$$
\|\nabla u\|_{L^{p}\left(B_{r} \cap \Omega\right)}^{p} \leqq C r^{\alpha}
$$

for some constants $\alpha>1$ and $C>0$ and for every ball $B_{r}$ with radius $r$. Condition (1.7) means that the bulk energy of the elastic configuration $u$ in a ball $B_{r}(x)$ is negligible with respect to the length of $\partial B_{r}(x)$ as $r$ goes to zero, uniformly in $x \in \bar{\Omega}$.

Our main result is the following theorem, which establishes that under the previous assumptions small cracks are not energetically convenient for the functional (1.3).

Theorem 1. Assume that $u$ admits only at most uniformly weak singularities in $\Omega$. Then there exists a critical length $l^{*}$ depending on $\Omega, f, k, \psi$, and $m$ such that for all $\Gamma \in \mathcal{K}_{m}(\bar{\Omega})$ with $\mathcal{H}^{1}(\Gamma)<l^{*}$ we have

$$
\int_{\Omega} f(x, \nabla u) \mathrm{d} x<\int_{\Omega} f\left(x, \nabla u_{\Gamma}\right) \mathrm{d} x+k \mathcal{H}^{1}(\Gamma) .
$$

We observe (see Remark 2) that this statement is equivalent to the local minimality of $u$ in (1.3), in the $L^{1}$ topology.

Let us briefly comment upon the assumption about the singularities of $u$ in Theorem 1. The minimality result is false if the elastic solution $u$ has strong singularities, namely if there exists $x \in \bar{\Omega}$ such that

$$
\limsup _{r \rightarrow 0} \frac{1}{r} \int_{B_{r}(x) \cap \bar{\Omega}}|\nabla u|^{p} \mathrm{~d} x=+\infty .
$$

In fact condition (1.9) ensures that it is energetically convenient to create a small crack $\Gamma:=\partial B_{r}(x)$ around $x$ : the surface energy needed to create such a crack is proportional to $r$, while the corresponding release of bulk energy is by (1.9) bigger than $r$ if $r$ is small enough.

The critical case when the right-hand side of (1.9) is a constant $0<C<\infty$ corresponds to the singularity appearing around the tip of the crack (see [21]). In this case the celebrated Irwin's formula states that the release of bulk energy per unit length along rectilinear increments of the crack is equal to the so-called mode III stress intensity factor $K_{I I I}$, which is proportional to $C$. In our class of cracks $\mathcal{K}_{m}(\bar{\Omega})$ we have that, if $C$ is small enough, then the release of bulk energy per unit length is less than $k$, and therefore our minimality result still holds, while it is false if $C$ is too large. We cannot fill the gap, and therefore we do not achieve a sharp Irwin-type formula in our class of cracks. 
In order to prove Theorem 1 we have to compare the asymptotic behavior of the release of bulk energy

$$
\int_{\Omega}\left[f(x, \nabla u)-f\left(x, \nabla u_{\Gamma}\right)\right] \mathrm{d} x
$$

with $\mathcal{H}^{1}(\Gamma)$ when $\mathcal{H}^{1}(\Gamma) \rightarrow 0$. In the literature there are many results in this direction considering particular sequences of infinitesimal cracks $\Gamma_{n}$, for instance when $\Gamma_{n}$ is the rescaled version of a fixed smooth curve $\Gamma$. An intuitive strategy to estimate (1.10) is to compute how much energy is required in order to make $u_{\Gamma}$ a good competitor for the minimum problem (1.6) without cracks, namely how much energy is required to heal the crack $\Gamma$.

This seems difficult for a generic crack in $\mathcal{K}_{m}(\bar{\Omega})$. So our strategy is to operate on the stress $\sigma:=\partial f(x, \nabla u)$ of the elastic solution. More precisely we prove the following key estimate (see (2.7))

$$
\int_{\Omega}\left[f(x, \nabla u)-f\left(x, \nabla u_{\Gamma}\right)\right] \mathrm{d} x \leqq \int_{\Omega}[\tau-\sigma] \cdot\left[\partial f^{*}(x, \tau)-\partial f^{*}(x, \sigma)\right] \mathrm{d} x,
$$

for all vector fields $\tau \in L^{q}\left(\Omega ; \mathbb{R}^{N}\right)\left(q=p^{\prime}:=\frac{p}{p-1}\right)$ such that

$$
\int_{\Omega} \tau \cdot \nabla v=0 \quad \text { for all } v \in \mathcal{A}(\Gamma) .
$$

Here $f^{*}$ is the convex conjugate of $f$, and $\mathcal{A}(\Gamma)$ is defined as

$$
\mathcal{A}(\Gamma):=\left\{v \in W^{1, p}(\Omega \backslash \Gamma): v=0 \text { on } \partial_{D} \Omega \backslash \Gamma\right\} .
$$

If $\Gamma$ is sufficiently regular, condition (1.12) implies that the vector field $\tau$ has zero divergence outside $\Gamma$, and $\tau(x)$ is tangent to $\Gamma$ for every $x \in \Gamma$.

The proof of Theorem 1 relies on the construction of a vector field $\tau$ satisfying (1.12) and such that

$$
\int_{\Omega}[\tau-\sigma] \cdot\left[\partial f^{*}(x, \tau)-\partial f^{*}(x, \sigma)\right] \mathrm{d} x<k \mathcal{H}^{1}(\Gamma) .
$$

We construct $\tau$ modifying the stress $\sigma$ which is divergence-free [as a consequence of Euler equation of problem (1.6)], but not tangent to $\Gamma$. First, we consider a neighborhood $U$ of $\Gamma$, and a cut-off function $\varphi$ such that $\varphi=0$ on $U$. Then $\varphi \sigma$ is null near $\Gamma$, and in particular it is tangent to $\Gamma$. Then we construct a vector field $\eta$ in such a way that $\eta=0$ on $U$ and

$$
\operatorname{div} \eta=-\operatorname{div}(\varphi \sigma) .
$$

We get that $\tau=\varphi \sigma+\eta$ is an admissible vector fields for inequality (1.11). Using the fact that $\Gamma \in \mathcal{K}_{m}(\bar{\Omega})$ and that $u$ has at most uniformly weak singularities, it is possible to choose $U, \varphi$, and $\eta$ in such a way that inequality (1.13) holds.

It turns out that the constraint (1.12) can be handled in an easier way than the constraint of being a gradient, and this is the reason why we work with the stress $\sigma$ instead of the strain $\nabla u$. 
Estimate (1.11) actually holds true in any dimension, and it turns out that our arguments work in any dimension provided that the crack $\Gamma$ belongs to the class

$$
\mathcal{K}^{C}(\bar{\Omega}):=\left\{\Gamma \subseteq \bar{\Omega}: \Gamma \text { is closed and } \operatorname{diam}(\Gamma) \leqq C \mathcal{H}^{N-1}(\Gamma)\right\},
$$

where $C$ is a fixed constant. This is certainly true in dimension two for the cracks in $\mathcal{K}_{1}(\bar{\Omega})$ [and in $\mathcal{K}_{m}(\bar{\Omega})$ up to a localization argument]. However, it also shows that the local minimality result of Theorem 1 remains valid in higher dimension, within the class $\mathcal{K}^{C}(\bar{\Omega})$ of cracks that are not needle-like: see Remark 7.

The minimality result holds also in the case of planar linearized elasticity, with a density of bulk energy involving the symmetrized gradient. This is considered in Section 4-while the "simpler" case of two-dimensional vectorial nonlinear elasticity is addressed in Remark 6.

A natural question that arises from Theorem 1 is whether the bound on the number of connected components is absolutely necessary for this result. Could it be possible to initiate fracture, in the framework of Griffith's theory, by nucleating infinitely many little cracks somewhere, possibly in a dense way? This is a very interesting point that remains open in general (we believe the answer is no). We can give a negative answer only in a very special case, of a scalar displacement in two dimensions, when everything is smooth enough. This is done in Appendix A, where we extend the local minimality result of Theorem 1 to the larger class of all one-dimensional rectifiable sets. This seems to be the most general class: however, there is a price to pay in order to handle such admissible cracks. First, we need to assume that $\nabla u$ is bounded and regular up to the boundary. In particular, we are not able to treat the case in which $u$ has weak singularities. Then, the method we employ is based on the maximum principle, which allows one to estimate the local opening of a crack with the global energy in a small ball surrounding the crack. It is therefore strictly scalar and bidimensional.

On the other hand, one could hope to show a priori a bound on the number of connected components of "good cracks" and thus be able to invoke, in all situations, Theorem 1. However, this seems quite difficult, as it is not even known for minimizers of the Mumford-Shah functional (1.3) (and is the last step towards a full proof of the Mumford-Shah conjecture in two dimensions [16]).

In fact, we would have expected, at the beginning of our study, that most of the questions we were asking could be addressed with techniques similar to the techniques introduced for the study of (almost, quasi, ... see for instance [16]) minimizers of the Mumford-Shah functional (1.3). We were not successful in this direction. In fact, it seems now quite different to us to study the properties of actual minimizers and to actually show that a given data is a local minimizer in some sense. Results exist that actually show that jumps sets of minimizers have their length/surface or density bounded from below (see, for instance, [4, 13, 16, 17, $25,28]$ ), but this does not really determine that in a given situation the energy will not decrease by adding a small fracture. The most straightforward strategy we had thought of, for instance, consisted of trying to study minimizers with a constraint $\mathcal{H}^{1}(K) \leqq \delta$ for $\delta$ small: however, the lower bounds shown in [16], if applicable for this constrained problem, would heavily depend on $\delta$ in a way that does not seem too explicit to us. Another approach would be to try to use in some recursive 
way excision lemmas à la MoRel and Solimini [25, 28, 31], but it is far from clear that this approach, if ever successful, would lead to a simpler proof than the one we present in the Appendix A (nor give any information on what happens in the presence of singularities).

The paper is organized as follows. In Section 2 we establish the main inequality (1.11). In Section 3 we prove the local minimality result in dimension 2 and its extensions to vector-valued displacements and to the $N$-dimensional case within the class of cracks given by (1.14). Section 4 addresses the case of planar twodimensional elasticity. The problem of crack initiation in quasistatic evolutions is addressed in Section 5, while the two-dimensional $S B V$-case without singularities is treated in Appendix A. In Appendix B we show how to obtain some uniform Poincaré and Poincaré-Korn type inequalities, used during the proofs of our main results in the construction of the competitor stress field $\tau$.

\section{The dual problem and the main estimate}

Let $\Omega$ be a bounded connected Lipschitz open set in $\mathbb{R}^{N}$, let $\partial_{D} \Omega \subseteq \partial \Omega$ be open in the relative topology, and let $\partial_{N} \Omega:=\partial \Omega \backslash \partial_{D} \Omega$. Let $f: \Omega \times \mathbb{R}^{N} \rightarrow \mathbb{R}$ be a Carathéodory function such that

$$
\begin{gathered}
\xi \rightarrow f(x, \xi) \text { is strictly convex and } C^{1} \text { for almost every } x \in \Omega, \\
f(x, 0)=0 \text { for almost every } x \in \Omega,
\end{gathered}
$$

and such that for almost every $x \in \Omega$ and for all $\xi \in \mathbb{R}^{N}$

$$
\alpha|\xi|^{p} \leqq f(x, \xi) \leqq \beta\left(|\xi|^{p}+1\right),
$$

where $\alpha, \beta>0$ and $1<p<+\infty$.

Given $\psi \in W^{1, p}(\Omega) \cap L^{\infty}(\Omega)$ and $\Gamma$ a closed set contained in $\bar{\Omega}$ (not necessarily with empty interior, nor with a bound on the number of its connected components), let us consider the minimization problem

$$
\min \left\{\int_{\Omega} f(x, \nabla u) \mathrm{d} x: u \in W^{1, p}(\Omega \backslash \Gamma), u=\psi \text { on } \partial_{D} \Omega \backslash \Gamma\right\} .
$$

In view of (2.2) and since $\psi \in W^{1, p}(\Omega) \cap L^{\infty}(\Omega)$, problem (2.4) is well posed.

Let us denote by $u_{\Gamma} \in W^{1, p}(\Omega \backslash \Gamma) \cap L^{\infty}(\Omega \backslash \Gamma)$ a minimizer of (2.4). Clearly $\nabla u_{\Gamma}$ is uniquely determined, while $u_{\Gamma}$ is determined up to a constant on each connected component of $\Omega \backslash \Gamma$ which does not touch $\partial_{D} \Omega$.

We denote by $u \in W^{1, p}(\Omega) \cap L^{\infty}(\Omega)$ the solution of (2.4) corresponding to $\Gamma=\emptyset$, and we refer to $u$ as the elastic solution. In the case $\Gamma$ is sufficiently regular, the Euler-Lagrange equation satisfied by $u_{\Gamma}$ is

$$
\begin{cases}\operatorname{div} \partial_{\xi} f\left(x, \nabla u_{\Gamma}\right)=0 & \text { on } \Omega \backslash \Gamma, \\ u=\psi & \text { on } \partial_{D} \Omega \backslash \Gamma, \\ \partial_{\xi} f\left(x, \nabla u_{\Gamma}\right) \cdot n=0 & \text { on } \partial_{N} \Omega \cup \partial \Gamma,\end{cases}
$$


where $n$ denotes the normal vector to $\partial_{N} \Omega \cup \partial \Gamma$. In the sequel, we will write $\partial f(x, \xi)$ for $\partial_{\xi} f(x, \xi)$.

Let us set

$$
\mathcal{A}(\Gamma):=\left\{v \in W^{1, p}(\Omega \backslash \Gamma): v=0 \text { on } \partial_{D} \Omega \backslash \Gamma\right\} .
$$

Let us denote by $f^{*}$ the convex conjugate of $f$ with respect to the second variable defined by

$$
f^{*}(x, \zeta):=\sup \left\{\zeta \cdot \xi-f(x, \xi): \xi \in \mathbb{R}^{N}\right\} .
$$

We refer the reader to [30] for the main properties of the conjugate function $f^{*}$. Notice that $f^{*}$ is of class $C^{1}$ since $f$ is strictly convex. The main result of this section is the following.

Theorem 2. Let $\Gamma$ be a closed subset of $\bar{\Omega}$, and let $\sigma:=\partial f(x, \nabla u)$ be the stress associated to the elastic configuration $u$. Then we have

$$
\begin{aligned}
& \int_{\Omega}\left[f(x, \nabla u)-f\left(x, \nabla u_{\Gamma}\right)\right] \mathrm{d} x \\
& \quad \leqq \int_{\Omega}[\tau-\sigma] \cdot\left[\partial f^{*}(x, \tau)-\partial f^{*}(x, \sigma)\right] \mathrm{d} x
\end{aligned}
$$

for all $\tau \in L^{q}\left(\Omega ; \mathbb{R}^{N}\right)\left(q=p^{\prime}:=\frac{p}{p-1}\right)$ such that

$$
\int_{\Omega} \tau \cdot \nabla v \mathrm{~d} x=0 \quad \text { for all } v \in \mathcal{A}(\Gamma) .
$$

Proof. For all $\eta \in L^{p}\left(\Omega ; \mathbb{R}^{N}\right)$ let us set

$$
\Phi(\eta):=\min _{w \in u+\mathcal{A}(\Gamma)} \int_{\Omega} f(x, \nabla w+\eta) \mathrm{d} x,
$$

where $\mathcal{A}(\Gamma)$ is defined in (2.6). Then the convex conjugate of $\Phi$ defined on $L^{q}\left(\Omega ; \mathbb{R}^{N}\right)$ has the form

$$
\begin{aligned}
\Phi^{*}(\tau) & :=\sup _{w, \eta} \int_{\Omega}[\tau \cdot \eta-f(x, \nabla w+\eta)] \mathrm{d} x \\
& =\sup _{w, \eta} \int_{\Omega}[\tau \cdot(\eta+\nabla w)-f(x, \nabla w+\eta)-\tau \cdot \nabla w] \mathrm{d} x \\
& =\int_{\Omega}\left[f^{*}(x, \tau)-\tau \cdot \nabla u\right] \mathrm{d} x+\sup _{v \in \mathcal{A}(\Gamma)} \int_{\Omega} \tau \cdot \nabla v \mathrm{~d} x
\end{aligned}
$$

We conclude that

$$
\Phi^{*}(\tau)= \begin{cases}\int_{\Omega}\left[f^{*}(x, \tau)-\tau \cdot \nabla u\right] \mathrm{d} x & \text { if } \tau \text { satisfies (2.8) } \\ +\infty & \text { otherwise. }\end{cases}
$$


Notice that $\Phi(0)=\Phi^{* *}(0)$ because $\Phi$ is weakly lower semicontinuous and

$$
\Phi(0)=\int_{\Omega} f\left(x, \nabla u_{\Gamma}\right) \mathrm{d} x<+\infty .
$$

(In fact, $\Phi$ is locally finite, hence locally Lipschitz.) Therefore we obtain

$$
-\int_{\Omega} f\left(x, \nabla u_{\Gamma}\right) \mathrm{d} x=-\Phi(0)=-\Phi^{* *}(0)=\min _{\tau} \Phi^{*}(\tau),
$$

so that by (2.11) we deduce

$$
-\int_{\Omega} f\left(x, \nabla u_{\Gamma}\right) \mathrm{d} x=\min _{\tau}\left\{\int_{\Omega}\left[f^{*}(x, \tau)-\tau \cdot \nabla u\right] \mathrm{d} x: \tau \text { satisfies }(2.8)\right\} .
$$

For all $\tau$ satisfying (2.8), we get

$$
\int_{\Omega}\left[f(x, \nabla u)-f\left(x, \nabla u_{\Gamma}\right)\right] \mathrm{d} x \leqq \int_{\Omega}\left[f(x, \nabla u)+f^{*}(x, \tau)-\tau \cdot \nabla u\right] \mathrm{d} x .
$$

Let $\sigma(x):=\partial f(x, \nabla u(x))$ be the stress of the elastic solution $u$. Since for almost every $x \in \Omega$

$$
f^{*}(x, \tau(x)) \leqq f^{*}(x, \sigma(x))+\partial f^{*}(x, \tau(x)) \cdot(\tau(x)-\sigma(x)),
$$

and

$$
f(x, \nabla u(x))+f^{*}(x, \sigma(x))=\nabla u(x) \cdot \sigma(x),
$$

and since $\nabla u(x)=\partial f^{*}(x, \sigma(x))$, we finally obtain our main estimate (2.7), so that the proof is concluded.

Remark 1. If $\Gamma$ is sufficiently regular, condition (2.8) implies that $\tau$ has zero divergence outside $\Gamma$, and $\tau(x)$ is tangent to $\partial \Gamma$ for every $x \in \partial \Gamma$.

\section{The minimality result in anti-plane elasticity}

In this section we prove that, under some assumptions on the elastic configuration $u$, small cracks are not convenient for the total energy

$$
\int_{\Omega} f(x, \nabla v) \mathrm{d} x+k \mathcal{H}^{1}(\Gamma),
$$

where $f$ is a Carathéodory function satisfying conditions (2.1), (2.2) and (2.3), and $k>0$.

Let us consider $\Omega$ bounded connected Lipschitz open subset of $\mathbb{R}^{2}$, and let $\partial_{D} \Omega \subseteq \partial \Omega$ be open in the relative topology and such that $\partial_{N} \Omega:=\partial \Omega \backslash \partial_{D} \Omega$ has a finite number of connected components. 
Let $m$ be a positive integer. The class of admissible cracks is given by

$$
\begin{gathered}
\mathcal{K}_{m}(\bar{\Omega}):=\{\Gamma \subseteq \bar{\Omega}: \Gamma \text { has at most } m \text { connected components and } \\
\left.\mathcal{H}^{1}(\Gamma)<+\infty\right\} .
\end{gathered}
$$

Given $\psi \in W^{1, p}(\Omega) \cap L^{\infty}(\Omega)$ and $\Gamma \in \mathcal{K}_{m}(\bar{\Omega})$, the displacement

$$
u_{\Gamma} \in W^{1, p}(\Omega \backslash \Gamma)
$$

associated to $\Gamma$ and $\psi$ is given by problem (2.4). We denote with $u$ the solution of (2.4) relative to $\Gamma=\emptyset$, and we refer to $u$ as the elastic solution.

The basic assumption on the elastic configuration $u$ involves the behavior of the energy concentration of the stress. We require that $u$ has at most uniformly weak singularities in $\Omega$, in the sense of the following definition.

Definition 1. We say that $u \in W^{1, p}(\Omega)$ has at most uniformly weak singularities in $A$, an open subset of $\Omega$, if there exist constants $1<\alpha<2$ and $C>0$ such that for every $x \in A$ and for every $r$ small (independent of $x$ )

$$
\int_{B_{r}(x) \cap \Omega}|\nabla u|^{p} \mathrm{~d} x \leqq C r^{\alpha} .
$$

As mentioned in the Introduction, the condition of at most uniformly weak singularities means that the bulk energy of the elastic configuration $u$ in a ball $B_{r}(x)$ is an infinitesimal of higher order than the length of $\partial B_{r}(x)$ as $r$ goes to zero, uniformly with respect to $x \in A$.

The main result of this section is the following theorem.

Theorem 3. Let the elastic solution $u$ of problem (2.4) have at most uniformly weak singularities in $\Omega$ according to Definition 1. Then there exists a critical length $l^{*}>0$ depending on $\Omega, m, f, k$, and $\psi$ such that for all $\Gamma \in \mathcal{K}_{m}(\bar{\Omega})$ with $\mathcal{H}^{1}(\Gamma)<l^{*}$ we have

$$
\int_{\Omega} f(x, \nabla u) \mathrm{d} x<\int_{\Omega} f\left(x, \nabla u_{\Gamma}\right) \mathrm{d} x+k \mathcal{H}^{1}(\Gamma),
$$

where $u_{\Gamma}$ is a minimum of (2.4).

Remark 2. (Local minimality in the $L^{1}$-topology) The minimality condition (3.4) implies that the elastic solution $u$ is a local minimum for the total energy (3.1) with respect to the $L^{1}$-topology. More precisely, for every sequence $\left(\Gamma_{h}\right)_{h \in \mathbb{N}}$ in $\mathcal{K}_{m}(\bar{\Omega})$ and for every $u_{h} \in W^{1, p}\left(\Omega \backslash \Gamma_{h}\right)$ with $u_{h}=\psi$ on $\partial_{D} \Omega \backslash \Gamma_{h}$ and $u_{h} \rightarrow u$ strongly in $L^{1}(\Omega)$, for $h$ large enough we have

$$
\int_{\Omega} f(x, \nabla u) \mathrm{d} x<\int_{\Omega} f\left(x, \nabla u_{h}\right) \mathrm{d} x+k \mathcal{H}^{1}\left(\Gamma_{h}\right) .
$$

In fact, it is not restrictive to assume that $\left(\nabla u_{h}\right)_{h \in \mathbb{N}}$ is bounded in $L^{p}\left(\Omega, \mathbb{R}^{2}\right)$ and that $\mathcal{H}^{1}\left(\Gamma_{h}\right) \leqq C$. By Ambrosio's lower semicontinuity theorem [2] we have

$$
\int_{\Omega} f(x, \nabla u) \mathrm{d} x \leqq \liminf _{h \rightarrow+\infty} \int_{\Omega} f\left(x, \nabla u_{h}\right) \mathrm{d} x .
$$


If the sequence $\left(\mathcal{H}^{1}\left(\Gamma_{h}\right)\right)_{h \in \mathbb{N}}$ is not infinitesimal, then (3.5) clearly holds. If $\mathcal{H}^{1}\left(\Gamma_{h}\right)$ $\rightarrow 0$, we have $\mathcal{H}^{1}\left(\Gamma_{h}\right) \leqq l^{*}$ for $h$ large enough, and hence (3.5) follows from (3.4).

Actually, using Ambrosio's compactness theorem [2], one can show that the local minimality in the $L^{1}$-topology and the minimality result of Theorem 3 are equivalent.

In order to prove Theorem 3 we will use the main estimate given by Theorem 2 . Our aim is to construct a vector field $\tau \in L^{q}\left(\Omega ; \mathbb{R}^{2}\right)$ which is an admissible competitor in (2.7) and which shows that the difference between the bulk energies of $u$ and $u_{\Gamma}$ is smaller than $k \mathcal{H}^{1}(\Gamma)$. In order to do so, we need some preliminary lemmas.

Lemma 1. Let $x \in \Omega$, and let $r>0$ be such that $B_{2 r}(x) \subseteq \Omega$. Let $\varphi$ be a smooth function with $0 \leqq \varphi \leqq 1, \varphi=0$ on $B_{r}(x), \varphi=1$ outside $B_{2 r}(x)$ and $\|\nabla \varphi\|_{\infty} \leqq \frac{2}{r}$. Then there exists $\eta \in L^{q}\left(\left(B_{2 r}(x) \backslash B_{r}(x)\right) ; \mathbb{R}^{2}\right)$ with $q:=p^{\prime}=p /(p-1)$ such that

$$
\begin{cases}\operatorname{div} \eta=-\operatorname{div}(\varphi \sigma) & \text { on } B_{2 r}(x) \backslash B_{r}(x) \\ \eta \cdot n=0 & \text { on } \partial\left(B_{2 r}(x) \backslash B_{r}(x)\right)\end{cases}
$$

and

$$
\int_{B_{2 r}(x) \backslash B_{r}(x)}|\eta|^{q} \mathrm{~d} x \leqq C \int_{B_{2 r}(x) \backslash B_{r}(x)}|\sigma|^{q} \mathrm{~d} x,
$$

where $\sigma:=\partial f(x, \nabla u)$ is the stress of the elastic solution $u, n$ is the outer normal to $\partial\left(B_{2 r}(x) \backslash B_{r}(x)\right)$, and $C$ is a constant independent of $r$.

Proof. Let us set $\eta:=|\nabla v|^{p-2} \nabla v$, where $v \in W^{1, p}\left(B_{2 r}(x) \backslash B_{r}(x)\right)$ satisfies the equation

$$
\begin{cases}\operatorname{div}\left(|\nabla v|^{p-2} \nabla v\right)=-\operatorname{div}(\varphi \sigma) & \text { in } B_{2 r}(x) \backslash B_{r}(x), \\ |\nabla v|^{p-2} \nabla v \cdot n=0 & \text { on } \partial\left(B_{2 r}(x) \backslash B_{r}(x)\right) .\end{cases}
$$

Notice that the equation is well posed because

$$
-\operatorname{div}(\varphi \sigma)=-\nabla \varphi \cdot \sigma \in L^{q}(\Omega)
$$

and

$$
\int_{B_{2 r}(x) \backslash B_{r}(x)} \operatorname{div}(\varphi \sigma) \mathrm{d} x=\int_{\partial B_{2 r}(x)} \sigma \cdot n \mathrm{~d} \mathcal{H}^{1}=0
$$

since $\sigma$ is divergence free in $\Omega$.

It remains to prove inequality (3.6). To this aim, note that we can always assume that $v$ has zero mean value, so that by the Poincaré inequality and by a rescaling argument, we have that there exists $C>0$ independent on $r$ such that

$$
\int_{B_{2 r}(x) \backslash B_{r}(x)}|v|^{p} \mathrm{~d} x \leqq C r^{p} \int_{B_{2 r}(x) \backslash B_{r}(x)}|\nabla v|^{p} \mathrm{~d} x .
$$


Recalling that $\|\nabla \varphi\|_{\infty} \leqq 2 / r$ and taking into account (3.8) and (3.9) we get

$$
\begin{aligned}
& \int_{B_{2 r}(x) \backslash B_{r}(x)}|\nabla v|^{p} \mathrm{~d} x \\
& \quad=\int_{B_{2 r}(x) \backslash B_{r}(x)} \nabla \varphi \cdot \sigma v \mathrm{~d} x \leqq\|\nabla \varphi \cdot \sigma\|_{L^{q}\left(B_{2 r}(x) \backslash B_{r}(x)\right)}\|v\|_{L^{p}\left(B_{2 r}(x) \backslash B_{r}(x)\right)} \\
& \quad \leqq \frac{1}{r}\|\sigma\|_{L^{q}\left(B_{2 r}(x) \backslash B_{r}(x), \mathbb{R}^{2}\right)} C^{1 / p} r\|\nabla v\|_{L^{p}\left(B_{2 r}(x) \backslash B_{r}(x), \mathbb{R}^{2}\right)} \\
& \quad=C^{1 / p}\|\sigma\|_{L^{q}\left(B_{2 r}(x) \backslash B_{r}(x), \mathbb{R}^{2}\right)}\|\nabla v\|_{L^{p}\left(B_{2 r}(x) \backslash B_{r}(x), \mathbb{R}^{2}\right)}
\end{aligned}
$$

so that

$$
\int_{B_{2 r}(x) \backslash B_{r}(x)}|\eta|^{q} \mathrm{~d} x=\int_{B_{2 r}(x) \backslash B_{r}(x)}|\nabla v|^{p} \mathrm{~d} x \leqq C^{q / p} \int_{B_{2 r}(x) \backslash B_{r}(x)}|\sigma|^{q} \mathrm{~d} x,
$$

and this concludes the proof.

Now we need to construct a suitable field $\eta$ also around points $x$ on the boundary of $\Omega$. Since $\Omega$ is Lipschitz, for every $x \in \partial \Omega$ we can find an orthogonal coordinate system $\left(x_{1}^{\prime}, x_{2}^{\prime}\right)$ with origin at $x, \varepsilon_{1}, \varepsilon_{2}>0$ and a Lipschitz function $g$ : $\left[-\varepsilon_{1}, \varepsilon_{1}\right] \rightarrow\left[-\varepsilon_{2}, \varepsilon_{2}\right]$ such that setting

$$
R_{r}(x):=\left\{\left(x_{1}^{\prime}, x_{2}^{\prime}\right):\left|x_{1}^{\prime}\right| \leqq r \varepsilon_{1},\left|x_{2}^{\prime}\right| \leqq r \varepsilon_{2}\right\}
$$

we have for $r$ small enough

$$
\Omega \cap R_{r}(x)=\left\{\left(x_{1}^{\prime}, x_{2}^{\prime}\right) \in R_{r}(x): x_{2}^{\prime} \geqq g\left(x_{1}^{\prime}\right)\right\} .
$$

Notice moreover that the Lipschitz constant $C_{g}$ of the function $g$ is determined only by $\Omega$, and that we can assume $C_{g} \varepsilon_{1}<\varepsilon_{2}$. Let us set

$$
A_{r}(x):=\left(R_{2 r}(x) \backslash R_{r}(x)\right) \cap \Omega .
$$

Lemma 2. Let $r>0$ be small enough (so that (3.11) holds everywhere on $\partial \Omega$ ). Let $x \in \partial \Omega$ such that one of the following three situations holds:

(1) $x \in \partial_{D} \Omega$ and $A_{r}(x) \cap \partial \Omega \subset \partial_{D} \Omega$;

(2) $x \in \operatorname{int}\left(\partial_{N} \Omega\right)$ and $A_{r}(x) \cap \partial \Omega \subset \partial_{N} \Omega$ (where int(.) indicates the interior relative to $\partial \Omega)$;

(3) $x \in \partial \Omega \backslash\left(\partial_{D} \Omega \cup \operatorname{int}\left(\partial_{N} \Omega\right)\right)$.

Let $\varphi$ be a smooth function with $0 \leqq \varphi \leqq 1, \varphi=0$ on $R_{r}(x), \varphi=1$ outside $R_{2 r}(x)$ and $\|\nabla \varphi\|_{\infty} \leqq \frac{2}{r}$. Then there exists $\eta \in L^{q}\left(A_{r}(x) ; \mathbb{R}^{2}\right)$ with $q:=p^{\prime}=p /(p-1)$ such that

$$
\begin{cases}\operatorname{div} \eta=-\operatorname{div}(\varphi \sigma) & \text { in } A_{r}(x) \\ \eta \cdot n=0 & \text { on } \partial A_{r}(x) \cap\left(\Omega \cup \partial_{N} \Omega\right)\end{cases}
$$

and

$$
\int_{A_{r}(x)}|\eta|^{q} \mathrm{~d} x \leqq C \int_{A_{r}(x)}|\sigma|^{q} \mathrm{~d} x
$$

where $\sigma:=\partial f(x, \nabla u)$ is the stress of the elastic solution $u, n$ is the outer normal to $\partial A_{r}(x) \cap\left(\Omega \cup \partial_{N} \Omega\right)$, and $C$ is a constant depending only on $\Omega$. 
We observe that only the points in $\partial \Omega$ at distance less than $r \sqrt{\varepsilon_{1}^{2}+\varepsilon_{2}^{2}}$ to $\partial \Omega \backslash$ $\left(\partial_{D} \Omega \cup \operatorname{int}\left(\partial_{N} \Omega\right)\right)$ might not fall into one of first two cases.

Proof. In all the three cases of the lemma, we will use the fact that the Poincare inequality holds in $W^{1, p}\left(A_{r}(x)\right)$ with a constant that rescales as $r$, that is, there exists a positive constant $C$ independent of $r$ such that

$$
\int_{A_{r}(x)}|v|^{p} \mathrm{~d} x \leqq C r^{p} \int_{A_{r}(x)}|\nabla v|^{p} \mathrm{~d} x
$$

for all $v \in W^{1, p}\left(A_{r}(x)\right)$ with

$$
\int_{A_{r}(x)} v=0 \text { or } v=0 \text { on } \partial_{D} \Omega \cap \partial A_{r}(x) .
$$

This can be seen by rescaling $A_{r}(x)$ with the transformation $T_{r}\left(x^{\prime}, y^{\prime}\right)=\left(\frac{x^{\prime}}{r}, \frac{y^{\prime}}{r}\right)$, and using Proposition 4 (see Appendix B) in the domains $\left\{T_{r}\left(A_{r}(x)\right)\right\}$ : it shows that the Poincare inequality holds in $T_{r}\left(A_{r}(x)\right)$ with a constant that is independent of $r$, and by rescaling we deduce that (3.13) holds.

In case (1), we can consider $\eta:=|\nabla v|^{p-2} \nabla v$, with $v \in W^{1, p}\left(A_{r}(x)\right)$ satisfying the equation

$$
\begin{cases}\operatorname{div}\left(|\nabla v|^{p-2} \nabla v\right)=-\operatorname{div}(\varphi \sigma) & \text { in } A_{r}(x), \\ v=0 & \text { on } \partial A_{r}(x) \cap \partial_{D} \Omega, \\ |\nabla v|^{p-2} \nabla v \cdot n=0 & \text { on } \partial A_{r}(x) \cap \Omega .\end{cases}
$$

Notice that $-\operatorname{div}(\varphi \sigma)=-\nabla \varphi \cdot \sigma(\sigma$ is divergence free $)$ and $\|\nabla \varphi\|_{\infty} \leqq \frac{1}{r}$. Taking into account the Poincaré inequality (3.13) we get

$$
\begin{aligned}
& \int_{A_{r}(x)}|\nabla v|^{p} \mathrm{~d} x \\
& \quad=\int_{A_{r}(x)} \nabla \varphi \cdot \sigma v \mathrm{~d} x \leqq\|\nabla \varphi \cdot \sigma\|_{L^{q}\left(A_{r}(x)\right)}\|v\|_{L^{p}\left(A_{r}(x)\right)} \\
& \quad \leqq \frac{1}{r}\|\sigma\|_{L^{q}\left(A_{r}(x), \mathbb{R}^{2}\right)} C^{1 / p} r\|\nabla v\|_{L^{p}\left(A_{r}(x), \mathbb{R}^{2}\right)} \\
& \quad=C^{1 / p}\|\sigma\|_{L^{q}\left(A_{r}(x), \mathbb{R}^{2}\right)}\|\nabla v\|_{L^{p}\left(A_{r}(x), \mathbb{R}^{2}\right)}
\end{aligned}
$$

so that

$$
\int_{A_{r}(x)}|\eta|^{q} \mathrm{~d} x=\int_{A_{r}(x)}|\nabla v|^{p} \mathrm{~d} x \leqq C^{q / p} \int_{A_{r}(x)}|\sigma|^{q} \mathrm{~d} x .
$$

Cases (2) and (3) can be treated as case (1) considering $\eta:=|\nabla v|^{p-2} \nabla v$ with $v \in W^{1, p}\left(A_{r}(x)\right)$ defined by the equations

$$
\begin{cases}\operatorname{div}\left(|\nabla v|^{p-2} \nabla v\right)=-\operatorname{div}(\varphi \sigma) & \text { in } A_{r}(x), \\ |\nabla v|^{p-2} \nabla v \cdot n=0 & \text { on } \partial A_{r}(x),\end{cases}
$$


and

$$
\begin{cases}\operatorname{div}\left(|\nabla v|^{p-2} \nabla v\right)=-\operatorname{div}(\varphi \sigma) & \text { in } A_{r}(x), \\ |\nabla v|^{p-2} \nabla v \cdot n=0 & \text { on } \partial A_{r}(x) \cap\left(\Omega \cup \partial_{N} \Omega\right), \\ v=0 & \text { on } \partial A_{r}(x) \cap \partial_{D} \Omega,\end{cases}
$$

respectively. Notice in particular that equation (3.15) is well posed since its righthand side has zero mean value, because $\sigma$ is divergence-free in $\Omega$, and $\sigma \cdot n=0$ on $\partial_{N} \Omega$ so that

$$
\int_{\Omega} \operatorname{div}(\varphi \sigma) \mathrm{d} x=\int_{A_{r}} \operatorname{div}(\varphi \sigma) \mathrm{d} x=\int_{\partial A_{r}} \sigma \cdot n \mathrm{~d} \mathcal{H}^{1}=0 .
$$

We are now in a position to prove our minimality result.

Proof. (Proof of Theorem 3) First of all, we claim that there exist at most $m$ open balls $B_{r_{1}}\left(x_{1}\right), \ldots, B_{r_{k}}\left(x_{k}\right), k \leqq m$ such that $\Gamma \subseteq \cup_{i} B_{r_{i}}\left(x_{i}\right)$,

$$
r_{i} \leqq C \mathcal{H}^{1}\left(B_{r_{i}}\left(x_{i}\right) \cap \Gamma\right),
$$

and

$$
B_{2 r_{i}}\left(x_{i}\right) \cap B_{2 r_{j}}\left(x_{j}\right)=\varnothing \text { for all } i \neq j,
$$

where $C$ depends only on $m$. In fact let us consider the decomposition of $\Gamma$ in its connected components, that is,

$$
\Gamma:=\Gamma_{1} \cup \cdots \cup \Gamma_{k}
$$

with $k \leqq m$. For all $i=1, \ldots, k$ let $B_{s_{i}}\left(y_{i}\right)$ be an open ball with $s_{i}=\mathcal{H}^{1}\left(\Gamma_{i}\right)$ and such that $\Gamma_{i} \subseteq B_{s_{i}}\left(y_{i}\right)$. If the balls $B_{2 s_{i}}\left(y_{i}\right)$ are disjoint, then the covering $\left\{B_{s_{i}}\left(y_{i}\right)\right\}_{i=1, \ldots, k}$ satisfies the claim. Otherwise we proceed in this way. Let us consider

$$
\mathcal{B}^{1}:=\bigcup_{i=1}^{k} B_{2 s_{i}}\left(y_{i}\right)
$$

and let $\mathcal{B}_{j}^{1}, j=1, \ldots, \tilde{k} \leqq k-1$ be its connected components. For all $j$, let $B_{\tau_{j}}\left(z_{j}\right)$ be an open ball with $\tau_{j}=\operatorname{diam}\left(\mathcal{B}_{j}^{1}\right)$ such that $\mathcal{B}_{j}^{1} \subseteq B_{\tau_{j}}\left(z_{j}\right)$. Again, if the balls $B_{2 \tau_{j}}\left(z_{j}\right)$ are disjoint, then the covering $\left\{B_{\tau_{j}}\left(z_{j}\right)\right\}_{j=1, \ldots, \tilde{k}}$ satisfies the claim. Otherwise we construct in a similar way as before the set $\mathcal{B}^{2}$ which has at most $k-2$ connected components. Clearly in at most $m$ steps we come up with at most $m$ balls satisfying the requirements of the claim.

Since $\Omega$ is Lipschitz, we have that, if $\mathcal{H}^{1}(\Gamma)$ is sufficiently small (depending on $\Omega$ ), we can assume that the balls $\left\{B_{r_{i}}\left(x_{i}\right)\right\}_{i=1, \ldots, k}$ intersecting $\partial \Omega$ can be replaced by rectangles $R_{S_{j}}\left(y_{j}\right)$ of the form (3.10) satisfying (3.11), centered at some point $y_{j}$ that falls into case (1), (2), or (3) of Lemma 2. More precisely, there exists a constant $C$ depending only on $\Omega$ and $m$, and there exist at most $m$ open balls $\left\{B_{r_{i}}\left(x_{i}\right)\right\}$ 
and at most $m$ rectangles $\left\{R_{s_{j}}\left(y_{j}\right)\right\}$ defined in (3.10) with $y_{j}$ falling into case (1), (2) or (3) of Lemma 2, such that $B_{2 r_{i}}\left(x_{i}\right) \subseteq \Omega$, and

$$
\begin{gathered}
\Gamma \subseteq \cup_{i, j} B_{r_{i}}\left(x_{i}\right) \cup R_{s_{j}}\left(y_{j}\right), \\
\operatorname{diam}\left(B_{r_{i}}\left(x_{i}\right)\right) \leqq C \mathcal{H}^{1}\left(B_{r_{i}}\left(x_{i}\right) \cap \Gamma\right), \\
\operatorname{diam}\left(R_{s_{j}}\left(y_{j}\right)\right) \leqq C \mathcal{H}^{1}\left(R_{s_{j}}\left(y_{j}\right) \cap \Gamma\right), \\
B_{2 r_{i}}\left(x_{i}\right) \cap B_{2 r_{j}}\left(x_{j}\right)=\emptyset, \\
R_{2 s_{i}}\left(y_{i}\right) \cap R_{2 s_{j}}\left(y_{j}\right)=\emptyset, \\
B_{2 r_{i}}\left(x_{i}\right) \cap R_{2 s_{j}}\left(y_{j}\right)=\emptyset \text { for all } i, j .
\end{gathered}
$$

Let $\varphi$ be a smooth function with

$$
\begin{aligned}
0 \leqq \varphi \leqq 1, \quad \varphi & =0 \text { on } \bigcup_{i, j}\left(B_{r_{i}}\left(x_{i}\right) \cup R_{s_{j}}\left(y_{j}\right)\right) \\
\varphi & =1 \text { outside } \bigcup_{i, j}\left(B_{2 r_{i}}\left(x_{i}\right) \cup R_{2 s_{j}}\left(y_{j}\right)\right) .
\end{aligned}
$$

Let us denote with $A_{r_{i}}\left(x_{i}\right)$ and $A_{s_{j}}\left(y_{j}\right)$ the sets $B_{2 r_{i}}\left(x_{i}\right) \backslash B_{r_{i}}\left(x_{i}\right)$ and $R_{2 s_{j}}\left(y_{j}\right) \backslash$ $R_{s_{j}}\left(y_{j}\right)$, respectively. Let

$$
\eta_{i} \in L^{q}\left(A_{r_{i}}\left(x_{i}\right) ; \mathbb{R}^{2}\right) \text { and } \eta_{j} \in L^{q}\left(A_{s_{j}}\left(y_{j}\right) ; \mathbb{R}^{2}\right)
$$

with $q:=p^{\prime}=p /(p-1)$ be the vector fields given by Lemmas 1 and 2 . Let us consider $\eta \in L^{q}\left(\Omega ; \mathbb{R}^{2}\right)$ defined as

$$
\eta:= \begin{cases}\eta_{i} & \text { in } A_{r_{i}}\left(x_{i}\right) \\ \eta_{j} & \text { in } A_{s_{j}}\left(y_{j}\right) \\ 0 & \text { otherwise }\end{cases}
$$

and let us set

$$
\tau:=\varphi \sigma+\eta,
$$

where $\sigma=\partial f(x, \nabla u)$ is the stress of the elastic solution $u$. By construction we have that

$$
\int_{\Omega} \tau \cdot \nabla v=0 \text { for all } v \in \mathcal{A}(\Gamma),
$$

where $\mathcal{A}(\Gamma)$ is as in (2.6). Moreover we have that for all $i, j$

$$
\int_{A_{h}}|\eta|^{q} \mathrm{~d} x \leqq C \int_{A_{h}}|\sigma|^{p} \mathrm{~d} x,
$$

where $A_{h}$ denotes one of the $A_{r_{i}}\left(x_{i}\right)$ 's or one of the $A_{s_{j}}\left(y_{j}\right)$ 's, and $C$ is a constant depending only on $\Omega$ and the bulk energy density $f$. 
In view of (3.18) we can put $\tau$ in inequality (2.7) getting

$$
\begin{aligned}
& \int_{\Omega}\left[f(x, \nabla u)-f\left(x, \nabla u_{\Gamma}\right)\right] \mathrm{d} x \\
& \quad \leq \sum_{h} \int_{A_{h}}[\tau-\sigma] \cdot\left[\partial f^{*}(x, \tau)-\partial f^{*}(x, \sigma)\right] \mathrm{d} x .
\end{aligned}
$$

Since $f^{*}$ is the convex conjugate of $f$, and since $f$ satisfies the growth conditions (2.3), we deduce that

$$
\left|f^{*}(x, \zeta)\right| \leqq C\left(|\zeta|^{q}+1\right)
$$

and

$$
\left|\partial_{\zeta} f^{*}(x, \zeta)\right| \leqq C\left(|\zeta|^{q-1}+1\right)
$$

We claim that for every $A_{h}$

$$
\int_{A_{h}}[\tau-\sigma] \cdot\left[\partial f^{*}(x, \tau)-\partial f^{*}(x, \sigma)\right] \leqq C \operatorname{diam}\left(A_{h}\right)^{\alpha},
$$

where $C$ is independent of $A_{h}$, and depends only on $m, \Omega$, and $f$. Here $\alpha>1$ is the exponent defining the weak singularities of $u$ (see Definition 1). From (3.20) and (3.19), taking into account (3.17) and the fact that $\alpha>1$, we obtain that there exists $l^{*}$ depending only on $\Omega, m, f$, and $k$ such that for every $\Gamma \in \mathcal{K}_{m}(\bar{\Omega})$ with $\mathcal{H}^{1}(\Gamma)<l^{*}$ we have

$$
\int_{\Omega}\left[f(x, \nabla u)-f\left(x, \nabla u_{\Gamma}\right)\right] \mathrm{d} x \leqq C \sum_{h} \operatorname{diam}\left(A_{h}\right)^{\alpha} \leqq C \mathcal{H}^{1}(\Gamma)^{\alpha}<k \mathcal{H}^{1}(\Gamma),
$$

so that the minimality result holds.

In order to conclude the proof, we need to show that claim (3.20) holds true. This can be seen making all the products and estimating each addend. Let us check the first one, the other ones being similar. We have

$$
\int_{A_{h}} \tau \cdot \partial f^{*}(x, \tau) \mathrm{d} x \leqq C \int_{A_{h}}(|\sigma|+|\eta|) \cdot\left(|\sigma|^{q-1}+|\eta|^{q-1}+1\right) \mathrm{d} x .
$$

Then, in view of Lemmas 1 and 2, since $u$ has at most uniformly weak singularities in $\Omega$ we get for $r$ small enough

$$
\begin{gathered}
\int_{A_{h}}|\sigma|^{q} \mathrm{~d} x \leqq C \int_{A_{h}}|\nabla u|^{p} \mathrm{~d} x+C\left|A_{h}\right| \leqq C \operatorname{diam}\left(A_{h}\right)^{\alpha} \\
\int_{A_{h}}|\eta|^{q} \mathrm{~d} x \leqq C \int_{A_{h}}|\sigma|^{p} \mathrm{~d} x \leqq C \operatorname{diam}\left(A_{h}\right)^{\alpha} \\
\int_{A_{h}}|\eta||\sigma|^{q-1} \mathrm{~d} x \leqq\left(\int_{A_{h}}|\eta|^{q} \mathrm{~d} x\right)^{\frac{1}{q}}\left(\int_{A_{h}}|\sigma|^{q} \mathrm{~d} x\right)^{\frac{1}{p}} \leqq C \operatorname{diam}\left(A_{h}\right)^{\alpha}
\end{gathered}
$$




$$
\begin{gathered}
\int_{A_{h}}|\eta|^{q-1}|\sigma| \mathrm{d} x \leqq\left(\int_{A_{h}}|\eta|^{q} \mathrm{~d} x\right)^{\frac{1}{p}}\left(\int_{A_{h}}|\sigma|^{q} \mathrm{~d} x\right)^{\frac{1}{q}} \leqq C \operatorname{diam}\left(A_{h}\right)^{\alpha}, \\
\int_{A_{h}}|\sigma| \mathrm{d} x \leqq C\left(\int_{A_{h}}|\sigma|^{q} \mathrm{~d} x\right)^{\frac{1}{q}} \operatorname{diam}\left(A_{h}\right)^{\frac{2}{p}} \leqq C \operatorname{diam}\left(A_{h}\right)^{\alpha}
\end{gathered}
$$

and

$$
\int_{A_{h}}|\eta| \mathrm{d} x \leqq C\left(\int_{A_{h}}|\eta|^{q} \mathrm{~d} x\right)^{\frac{1}{q}} \operatorname{diam}\left(A_{h}\right)^{\frac{2}{p}} \leqq C \operatorname{diam}\left(A_{h}\right)^{\alpha} .
$$

Summing up we obtain that (3.20) holds.

Remark 3. Notice that the arguments of the previous proof also work in the case in which the elastic solution $u$ has critical singularities, that is, in the case $\alpha=1$, provided that there exists $\varepsilon>0$ and $\delta$ sufficiently small (depending only on $\Omega, m$, $f$ and $k$ ), such that

$$
\int_{B_{r}(x) \cap \bar{\Omega}}|\nabla u|^{p} \mathrm{~d} x \leqq \delta r \quad \text { for every } r<\varepsilon .
$$

Using the same arguments of the proof of Theorem 3, we can easily deduce the following localized version of the minimality result.

Proposition 1. Let the elastic solution u of problem (2.4) have at most uniformly weak singularities in A, where A is an open subset of $\Omega$. Then there exists a critical length $l^{*}>0$ depending on $A, m, f$, and $k$ such that for all $\Gamma \in \mathcal{K}_{m}(\bar{\Omega})$ with $\Gamma \subseteq \bar{A}$ and $\mathcal{H}^{1}(\Gamma)<l^{*}$ we have

$$
\int_{\Omega} f(x, \nabla u) \mathrm{d} x<\int_{\Omega} f\left(x, \nabla u_{\Gamma}\right) \mathrm{d} x+k \mathcal{H}^{1}(\Gamma),
$$

where $u_{\Gamma}$ is a minimum of (2.4).

Remark 4. (The case of strong singularities) Theorem 3 is false if the elastic solution $u$ has strong singularities in $\Omega$, that is, there exists $x \in \bar{\Omega}$ such that

$$
\limsup _{r \rightarrow 0} \frac{1}{r} \int_{B_{r}(x) \cap \Omega}|\nabla u|^{p} \mathrm{~d} x=+\infty .
$$

In fact if such a point $x$ exists, then the pair $\left(v_{r}, \partial B_{r}(x) \cap \bar{\Omega}\right)$, where $v_{r}$ is defined as

$$
v_{r}(y):= \begin{cases}u(y) & \text { in } \bar{\Omega} \backslash B_{r}(x), \\ 0 & \text { in } \bar{\Omega} \cap B_{r}(x)\end{cases}
$$

is energetically more convenient with respect to $u$ for $r$ small. Note also that this example needs the right-hand side of (3.21) to be just greater than $\frac{2 \pi}{k}$. 
The previous remark and Proposition 1 seem to suggest that, in the presence of strong singularities, energetically convenient small cracks prefer to stay near the singular points. Let us prove that this intuition is indeed true in the case in which $u$ has only one point of strong singularity $x$.

For every $l>0$ let $\Gamma_{l} \in \mathcal{K}_{m}(\bar{\Omega})$ be such that $\left(u_{\Gamma_{l}}, \Gamma_{l}\right)$ minimizes the total energy (3.1) among all pairs $\left(u_{\Gamma}, \Gamma\right)$ with $\Gamma \in \mathcal{K}_{m}(\bar{\Omega})$ and $\mathcal{H}^{1}(\Gamma) \leqq l$. The existence of $\Gamma_{l}$ can be proved using the direct method of the Calculus of Variations in view of the lower semicontinuity of the $\mathcal{H}^{1}$-measure with respect to Hausdorff converging sequences in $\mathcal{K}_{m}(\bar{\Omega})$ given by Gołab's Theorem (see, for example, [14] for details).

Notice that $\Gamma_{l} \neq \varnothing$ for $l$ small because the pair $\left(v_{l}, \partial B_{l}\right)$, where $v_{l}$ is defined in (3.22), is energetically more convenient than the elastic solution. Moreover, for every $r>0$, we have that $\Gamma_{l} \cap B_{r}(x) \neq \emptyset$ when $l$ is small enough because otherwise, in view of Proposition $1,\left(u_{\Gamma_{l}}, \Gamma_{l}\right)$ would not be energetically more convenient than the elastic solution. So we deduce that the following proposition holds.

Proposition 2. Assume that $x \in \Omega$ is a point of strong singularity for the elastic solution $u$, and that $u$ has at most uniformly weak singularities in $\Omega \backslash B_{r}(x)$ for every $r>0$. Then for every neighborhood $U$ of $x$, if $l$ is small enough we have $\Gamma_{l} \neq \emptyset$, and $\Gamma_{l} \cap U \neq \emptyset$.

Remark 5. (Singularities in materials) Theorem 3 and Remark 4 show that the quantity

$$
\int_{\Omega \cap B_{r}(x)}|\nabla u|^{p} \mathrm{~d} x
$$

where $u$ is a solution of

$$
\begin{cases}\operatorname{div} \partial_{\xi} f(x, \nabla u)=0 & \text { in } \Omega \\ u=\psi & \text { on } \partial_{D} \Omega\end{cases}
$$

determines the feasibility of the appearance of small cracks in $\bar{\Omega}$ when imposing a boundary datum $\psi$. By Theorem 3, small cracks are not energetically convenient if $u$ has at most uniformly weak singularities in $\Omega$ according to Definition 1 . This is certainly the case if $\nabla u$ has a summability sufficiently higher than $p$, as one can check by means of Hölder inequality. In fact we have

$$
\begin{aligned}
\int_{B_{r}(x)}|\nabla u|^{p} \mathrm{~d} x & \leqq\left(\int_{B_{r}(x)}|\nabla u|^{q} \mathrm{~d} x\right)^{p / q}\left|B_{r}(x)\right|^{(q-p) / q} \\
& \leqq\left(\int_{\Omega}|\nabla u|^{q} \mathrm{~d} x\right)^{p / q} r^{2(q-p) / q} .
\end{aligned}
$$

We deduce that $u$ has at most weak singularities in $\Omega$ if $\nabla u \in L^{q}\left(\Omega ; \mathbb{R}^{2}\right)$ with $q>2 p$.

It is well known that, if $\Omega, \psi$, and $f$ are sufficiently regular, then the solution $u$ of (3.24) is regular, so that $u$ has at most uniformly weak singularities in 
$\Omega$. However the assumption of regularity of $f$ with respect to the variable $x$ is not suitable for applications to continuum mechanics, since discontinuity in the variable $x$ models the important case of composite materials.

Several papers in the literature address the issue of higher integrability properties of the gradient of solutions of (3.24) without continuity assumptions on $x$. Usually, the behavior of $\nabla u$ is studied on compactly contained open subsets of $\Omega$, and this is the natural price to pay in order to concentrate on properties depending only on the material (that is, on the bulk energy density $f$ ) and not on the boundary datum.

In the fundamental paper [26] due to Meyers (and based on some ideas by BOYARSKI on higher integrability for quasiconformal mappings in dimension two, see $[8,9])$, it is proved in particular the following result. Let $A$ be an $N \times N$ symmetric matrix with eigenvalues between $K^{-1}$ and $K(K \geqq 1)$. Then the gradient of the solution of the linear elliptic partial differential equation (PDE)

$$
\begin{cases}\operatorname{div}(A(x) \nabla u)=0 & \text { in } \Omega \\ u=\psi & \text { on } \partial_{D} \Omega\end{cases}
$$

belongs to $L_{l o c}^{q}(\Omega)$ for some $q>2$. Moreover in the same paper it is conjectured that the optimal integrability exponent $q(K)$ is equal to $2 K /(K-1)$. This conjecture in particular implies that

$$
q(K) \rightarrow+\infty \text { for } K \rightarrow 1 \text { and } q(K) \rightarrow 2 \text { for } K \rightarrow+\infty
$$

The long-standing conjecture for the optimal exponent $q(K)$ in the context of planar quasiconformal mappings was solved by Astala in [6]. Then LeOnetTI and NESI [22] proved the conjecture in the context of PDEs: any solution of (3.25) has gradient in $L_{\text {loc }}^{q}(\Omega)$ for every $q<2 K /(K-1)$.

In the two-dimensional setting required by Theorem 3 , we conclude that, in order to guarantee that $u$ has at most uniformly weak singularities in compactly contained open subsets of $\Omega$, it suffices that $K<2$. On the other hand, a famous example due to Meyers [26] shows that for every $K \geqq 2$ we can find materials which exhibit strong singularities inside the body. In fact, assuming that the origin belongs to $\Omega$ we can consider

$$
A(x):=K n \otimes n+\frac{1}{K} \tau \otimes \tau,
$$

where $n:=\frac{x}{|x|}, \tau$ is obtained from $n$ through a rotation of 90 degrees counterclockwise, and $a \otimes b$ denotes the matrix with coefficients $(a \otimes b)_{i j}=a_{i} b_{j}$. Then it is easy to see that the solution of

$$
\begin{cases}\operatorname{div}(A(x) \nabla u)=0 & \text { in } \Omega \\ u(x)=x_{1} & \text { on } \partial \Omega\end{cases}
$$

is given by

$$
u(x):=|x|^{K^{-1}-1} x_{1} .
$$


A simple computation shows that if $K \geqq 2$, the origin is a point of strong singularity for $u$.

Higher integrability results for the gradient in the case of composite materials composed of a finite number of phases with some geometrical constraints can be found in Li-Vogelius [24] and in Li-NiRENBERG [23] (for the case of systems).

Higher integrability results in the case of nonlinear PDEs can be found in CAffarelli and Peral [11]. They prove [11, Theorem C] that, under mild assumptions on $f$, for any fixed $q>p$ there exists $\varepsilon=\varepsilon(q)>0$ such that if

$$
\left.|| \xi\right|^{p-2} \xi-\left.\partial_{\xi} f(x, \xi)|\leqq \varepsilon| \xi\right|^{p-1}
$$

(that is, $\partial_{\xi} f$ is sufficiently close to the $p$-Laplacian operator), then every solution $u \in W^{1, p}(\Omega)$ also belongs to $W_{\text {loc }}^{1, q}(\Omega)$. In particular if $\varepsilon$ is small enough, $u$ has at most uniformly weak singularities on every compactly contained open subset of $\Omega$.

Remark 6. (The vectorial two-dimensional case) The minimality result given by Theorem 3 holds also in the case of $\mathbb{R}^{M}$-valued displacements provided that we choose them in the Deny-Lions space

$$
L^{1, p}\left(\Omega \backslash \Gamma ; \mathbb{R}^{M}\right):=\left\{v \in W_{\text {loc }}^{1, p}\left(\Omega \backslash \Gamma, \mathbb{R}^{M}\right): \nabla v \in L^{p}\left(\Omega \backslash \Gamma ; M^{M \times 2}\right)\right\} .
$$

We have that $W^{1, p}\left(\Omega \backslash \Gamma, \mathbb{R}^{M}\right) \subseteq L^{1, p}\left(\Omega \backslash \Gamma ; \mathbb{R}^{M}\right)$, and the two spaces are equal if $\Omega \backslash \Gamma$ is sufficiently regular (for example, a union of a finite number of Lipschitz domains). A notion of trace for functions in $L^{1, p}\left(\Omega \backslash \Gamma ; \mathbb{R}^{M}\right)$ near the points of $\partial_{D} \Omega \backslash \Gamma$ is well defined, so that, given $\psi \in W^{1, p}\left(\Omega, \mathbb{R}^{M}\right)$ and $\Gamma \in \mathcal{K}_{m}(\bar{\Omega})$, the displacement $u_{\Gamma}$ is a solution of the minimum problem

$$
\min \left\{\int_{\Omega} f(x, \nabla u) \mathrm{d} x: u \in L^{1, p}\left(\Omega \backslash \Gamma ; \mathbb{R}^{M}\right), u=\psi \text { on } \partial_{D} \Omega \backslash \Gamma\right\} .
$$

(Notice that in this vectorial setting the maximum principle does not hold, so that only a control on the gradient is available and this is why Deny-Lions spaces are required.) Since Definition 1 relies only on the behavior of $\nabla u$, it turns out that the notion of uniformly weak singularities is well defined in the context of $L^{1, p}$-spaces.

The minimality result in the vectorial setting follows because estimate (2.7) still holds provided we set

$$
\mathcal{A}(\Gamma):=\left\{v \in L^{1, p}\left(\Omega \backslash \Gamma ; \mathbb{R}^{M}\right): v=0 \text { on } \partial_{D} \Omega \backslash \Gamma\right\},
$$

and the constructions of Lemmas 1 and 2 can easily be adapted to the case of matrix-valued vector fields.

Remark 7. (The $N$-dimensional case) Let us consider the case $\Omega \subseteq \mathbb{R}^{N}$ with $N \geqq 3$. The two-dimensional setting is employed in the proof of Theorem 3 only to ensure the existence of a covering of the crack $\Gamma$ which satisfies conditions (3.17). In the case $\Gamma$ is connected, the covering condition can be reduced to the existence 
of an open set $A$ (a ball if $\Gamma$ is well inside $\Omega$, or a rectangle if it is near the boundary) such that $\Gamma \subseteq A$ and

$$
\operatorname{diam}(A) \leqq C \mathcal{H}^{N-1}(\Gamma),
$$

where $C$ is a given constant. This inequality is not implied by connectedness in dimension $N \geqq 3$, because needle-like cracks can have very small $\mathcal{H}^{N-1}$-measure and large diameter. So the machinery of the proof of Theorem 3 can be employed in dimension $N \geqq 3$ if we restrict ourselves to a class of admissible cracks which excludes the elongated ones. Namely we can consider the family

$$
\mathcal{K}^{C}(\bar{\Omega}):=\left\{\Gamma \subseteq \bar{\Omega}: \Gamma \text { is closed and } \operatorname{diam}(\Gamma) \leqq C \mathcal{H}^{N-1}(\Gamma)\right\}
$$

where $C$ is a given constant. The displacement $u_{\Gamma}$ relative to a crack $\Gamma \in \mathcal{K}^{C}(\bar{\Omega})$ and the boundary datum $\psi \in W^{1, p}\left(\Omega, \mathbb{R}^{M}\right)$, with $M \geqq 1$, is again given by problem

$$
\min \left\{\int_{\Omega} f(x, \nabla u) \mathrm{d} x: u \in L^{1, p}\left(\Omega \backslash \Gamma ; \mathbb{R}^{M}\right), u=\psi \text { on } \partial_{D} \Omega \backslash \Gamma\right\},
$$

where the space $L^{1, p}\left(\Omega \backslash \Gamma ; \mathbb{R}^{M}\right)$ is defined in (3.26).

The notion of uniformly weak singularities can be rephrased in the $N$-dimensional setting in the following way: we say that $u \in L^{1, p}\left(\Omega ; \mathbb{R}^{N}\right)$ has at most uniformly weak singularities in $\Omega$ if there exist constants $N-1<\alpha \leqq N$ and $C>0$ such that for every $x \in \bar{\Omega}$ and for $r$ small enough

$$
\int_{B_{r}(x) \cap \Omega}|\nabla u|^{p} \mathrm{~d} x \leqq C r^{\alpha} .
$$

The intuitive meaning of condition (3.30) is the same as in the two-dimensional setting, namely that the bulk energy of $u$ inside a ball of center $x$ and radius $r$ is asymptotically negligible for $r \rightarrow 0$ and uniformly in $x$ with respect to the surface of the ball.

\section{The minimality result in linearized elasticity}

We briefly show in this section that the results obtained up to now are also true if the bulk energy $f(x, \nabla u)$ is replaced with a quadratic linearized elasticity energy

$$
C(x) e(u): e(u)=C_{i, j, k, l}(x) e(u)_{i, j} e(u)_{k, l},
$$

that is a positive-definite quadratic form of the symmetrized gradient

$$
e(u):=\left(\nabla u+(\nabla u)^{\mathrm{T}}\right) / 2
$$

of the displacement $u: \Omega \rightarrow \mathbb{R}^{N}$. As before, the main conclusions will be drawn in dimension 2 (Theorem 3 or rather, since $u$ is vectorial, the result of Remark 6), whereas in higher dimension the same restrictions of Remark 7 will apply. 
Let us consider in $\Omega$ a measurable function $C$, such that for any $x \in \Omega, C(x)$ is an $N \times N \times N \times N$ fourth-order tensor that defines a positive-definite quadratic form on the vector space of symmetric $N \times N$ matrices, that we denote by $\mathcal{S}^{N \times N}$. We assume that for any $\xi \in \mathcal{S}^{N \times N}$ and almost every $x \in \Omega$, it holds that

$$
\lambda|\xi|^{2} \leqq C(x) \xi: \xi \leqq \Lambda|\xi|^{2},
$$

where $\xi: \eta=\operatorname{Tr}\left(\xi \eta^{T}\right)=\xi_{i, j} \eta_{i, j}$ and $|\xi|^{2}=\xi: \xi$ is the standard Euclidean (Frobenius) norm.

Given $\Gamma \subset \bar{\Omega}$ a compact one-dimensional fracture, the space of admissible displacements with finite energy will be the space of measurable displacements $u: \Omega \rightarrow \mathbb{R}^{N}$ whose symmetrized distributional gradient in $\Omega \backslash \Gamma$, denoted by $e(u)$, is in $L^{2}\left(\Omega \backslash \Gamma ; \mathcal{S}^{N \times N}\right)$, and that satisfy in some sense $u=\psi$ on $\partial_{D} \Omega \backslash \Gamma$. Thanks to Korn's inequality, it is known that such a displacement belongs in fact to $H_{\text {loc }}^{1}(\Omega \backslash \Gamma)$, and since we have assumed that the boundary of $\Omega$ is Lipschitz, we also have that $u \in H^{1}(\Omega \cap B)$ for any ball $B$ with $\bar{B} \cap \Gamma=\emptyset$, so that the trace of $u$ on $\partial \Omega \backslash \Gamma$ is well defined. As in Section 6, we introduce the space

$$
L D^{1,2}(\Omega \backslash \Gamma):=\left\{u \in H_{\mathrm{loc}}^{1}\left(\Omega \backslash \Gamma ; \mathbb{R}^{N}\right): e(u) \in L^{2}\left(\Omega \backslash \Gamma ; \mathcal{S}^{N \times N}\right)\right\}
$$

and for $\Gamma$ a closed subset of $\bar{\Omega}$, the displacement $u_{\Gamma}$ is given by

$$
\min \left\{\int_{\Omega} C(x) e(u): e(u) \mathrm{d} x: u \in L D^{1,2}(\Omega \backslash \Gamma), u=\psi \text { on } \partial_{D} \Omega \backslash \Gamma\right\} .
$$

We denote by $u$ the solution in the non-cracked domain. The set of admissible variations is now

$$
\mathcal{A}(\Gamma):=\left\{v \in L D^{1,2}(\Omega \backslash \Gamma): v=0 \text { on } \partial_{D} \Omega \backslash \Gamma\right\} .
$$

The proof of Theorem 2 can be reproduced in this situation, yielding the estimate (we assume $|\Gamma|=0$ )

$$
\begin{aligned}
& \int_{\Omega}\left[C(x) e(u): e(u)-C(x) e\left(u_{\Gamma}\right): e\left(u_{\Gamma}\right)\right] \mathrm{d} x \\
& \quad \leqq 2 \int_{\Omega}[\tau-\sigma]:\left[C(x)^{-1}(\tau-\sigma)\right] \mathrm{d} x
\end{aligned}
$$

with $\sigma(x):=C(x) e(u)(x)$ and $\tau \in L^{2}\left(\Omega ; \mathcal{S}^{N \times N}\right)$ is any stress field compatible with the variations in $\mathcal{A}(\Gamma)$, that is, such that

$$
\int_{\Omega \backslash \Gamma} \tau: e(v) \mathrm{d} x=0
$$

for any $v \in \mathcal{A}(\Gamma)$. The last estimate, together with (4.1), yields

$$
\int_{\Omega}\left[C(x) e(u): e(u)-C(x) e\left(u_{\Gamma}\right): e\left(u_{\Gamma}\right)\right] \mathrm{d} x \leqq \frac{2}{\lambda} \int_{\Omega}|\tau-\sigma|^{2} \mathrm{~d} x,
$$

for any $\tau$ satisfying (4.2). 
In order to prove Theorem 3 in this setting, we form exactly the same construction as before. We build a $\tau$ from $\sigma$ by letting $\tau=\sigma$ in $\Omega$ except in a finite union of balls or rectangles $B_{2 r_{i}}\left(x_{i}\right)$ or $R_{2 s_{j}}\left(y_{j}\right)$ (cf. the proof of Theorem 3 pp. 322 and following). Inside the smaller balls/rectangles $B_{r_{i}}\left(x_{i}\right)$ and $R_{s_{j}}\left(y_{j}\right)$, we choose $\tau=0$, and in each crown $A_{r_{i}}\left(x_{i}\right)=B_{2 r_{i}}\left(x_{i}\right) \backslash B_{r_{i}}\left(x_{i}\right)$ or $A_{s_{j}}\left(y_{j}\right)=R_{2 s_{j}}\left(y_{j}\right) \backslash R_{s_{j}}\left(y_{j}\right)$, $\tau$ is of the form: $\tau=\varphi \sigma+\eta$, where $\varphi$ is the appropriate cut-off function.

Again, to achieve (4.2), one needs to choose $\eta$ in an appropriate way. An additional difficulty here follows from the fact that $\eta(x)$ has to be almost everywhere an $N \times N$ symmetric matrix. In order to find a suitable $\eta$, one needs to replace problems (3.7), (3.14), (3.15) or (3.16) by the appropriate variant.

The right way to do it is obviously to solve the vectorial equation

$$
\operatorname{div} e(v)=-\operatorname{div}(\varphi \sigma)
$$

in the appropriate domain, and to replace the Neumann boundary condition, when present, with the corresponding condition $e(v) \cdot n=0$. We then set $\eta=e(v)$ in each crown.

We get an estimate on $\int_{\Omega}|\tau-\sigma|^{2} \mathrm{~d} x$ from standard estimates on $e(v)$, that will follow from appropriate Poincaré-Korn inequalities. For instance, one shows (see Appendix B for details) that

$$
\int_{A_{r}(x)}|v|^{2} \mathrm{~d} x \leqq C r^{2} \int_{A_{r}(x)}|e(v)|^{2} \mathrm{~d} x
$$

for any $v \in H^{1}\left(A_{r}(x) ; \mathbb{R}^{N}\right)$ with

$$
\begin{gathered}
\left(\int_{A_{r}(x)} v(y) \mathrm{d} y=0 \text { and } \int_{A_{r}(x)} y \times v(y) \mathrm{d} y=0\right), \\
\text { or } v=0 \text { on } \partial_{D} \Omega \cap \partial A_{r}(x) .
\end{gathered}
$$

The first set of conditions ensure that the average rigid motion of $v$ vanishes in $A_{r}(x)$. Rigid motions, of the form $x \mapsto K x+p$ with $K$ antisymmetric, are the kernel of the symmetrized gradient (in any connected domain).

Multiplying (4.4) by $v$ and integrating by parts, we find that

$$
\begin{aligned}
& \int_{A_{r}(x)}|e(v)|^{2} \mathrm{~d} x \\
& \quad=-\int_{A_{r}(x)} \sigma:(\nabla \varphi \otimes v(x)) \mathrm{d} x \\
& \quad \leqq\|\sigma\|_{L^{2}\left(A_{r}(x)\right)}\|\nabla \varphi\|_{L^{\infty}\left(A_{r}(x)\right)}\left(\sqrt{C} r\|e(v)\|_{L^{2}\left(A_{r}(x)\right)}\right),
\end{aligned}
$$

where we have used (4.5). Since $\|\nabla \varphi\|_{L^{\infty}\left(A_{r}(x)\right)} \leqq 2 / r$ and $\eta=e(v)$, we deduce that

$$
\int_{A_{r}(x)}|\eta(x)|^{2} \mathrm{~d} x \leqq C \int_{A_{r}(x)}|\sigma(x)|^{2} \mathrm{~d} x
$$

for some constant $C$ that does not depend on $x$ or $r$. We conclude as in the proof of Theorem 3. 


\section{Qualitative properties of crack initiation}

In this section we use the results of Section 3 to address the problem of crack initiation in elastic bodies. We restrict our analysis to the case of antiplane elasticity. In view of the minimality result of Section 4, the same conclusions hold also for the case of planar linearized elasticity.

First of all we consider the classical theory of Griffith concerning quasistatic crack propagation, and we prove that it cannot explain the formation of a crack in an elastic body $\Omega$ without singularities within the class $\mathcal{K}_{m}(\bar{\Omega})$ of closed sets with a finite number of connected components, which is much richer than the family of smooth curves usually considered in the mechanical literature.

In the second part of the section, we remove the assumption, implicit in Griffith's theory, that the crack growth is progressive, namely that the length of the crack is continuous in time. Inspired by the variational theory of quasistatic crack evolution proposed by FRANCFORT and MARIGO in [20], we replace Griffith's equilibrium condition with a static equilibrium condition and an energy balance. The static equilibrium condition is a unilateral minimality property which states that, during the crack evolution, the total energy is minimal among all configurations with larger cracks (so that discontinuities of the crack's length are allowed). The energy balance requires that the total energy of the system evolves in relation with the power of external loads in such a way that no dissipation occurs (except the surface energy spent to enlarge the crack).

Within this framework, we prove that a crack appears immediately at a point of strong singularity for the body. Moreover we prove that, if the body has at most uniformly weak singularities, then it deforms elastically until a critical time $t_{i}$ after which a "big" crack $\Gamma(t)$ appears. These results have been established by FrancFort and MARIGO in [20, Proposition 4.19, point (ii)] under the assumptions that the crack $\Gamma(t)$ is union of $m$ fixed curves $\left\{\gamma_{i}(t)\right\}_{i=1, \ldots, m}$ which can be parameterized by arc length. Thanks to our local minimality result (Theorem 3 ), we prove these facts removing the restrictions on the path of the crack.

The mathematical setting we consider is that of Section 3. Namely $\Omega$ is a bounded Lipschitz open set in $\mathbb{R}^{2}, \partial_{D} \Omega \subseteq \partial \Omega$ is open in the relative topology, and $\partial_{N} \Omega:=\partial \Omega \backslash \partial_{D} \Omega$ is composed of a finite number of connected components. The family of admissible cracks is given by the class $\mathcal{K}_{m}(\bar{\Omega})$ of closed subsets of $\bar{\Omega}$ with at most $m$ connected components and with finite length, while the class of admissible displacements relative to a crack $\Gamma$ is given by $W^{1, p}(\Omega \backslash \Gamma)$ with $p \in] 1,+\infty[$. The total energy is given by

$$
\mathcal{E}(u, \Gamma):=\int_{\Omega} f(x, \nabla u) \mathrm{d} x+k \mathcal{H}^{1}(\Gamma),
$$

where $f$ is a Carathéodory function satisfying (2.1), (2.2) and (2.3), and $k>0$.

\subsection{Crack initiation and Griffith's theory}

Let $\Gamma_{0}$ be a crack inside $\bar{\Omega}$ of length $l_{0}$, and suppose that a boundary displacement $\psi$ is assigned on $\partial_{D} \Omega \backslash \Gamma$. According to Griffith theory, $\Gamma_{0}$ is in equilibrium if, 
taking any family of increasing cracks $\Gamma_{l}$ containing $\Gamma_{0}$ with length $l_{0}+l$, then

$$
\limsup _{l \rightarrow 0^{+}} \frac{\mathcal{W}\left(l_{0}\right)-\mathcal{W}\left(l_{0}+l\right)}{l} \leqq k,
$$

where $\mathcal{W}\left(l_{0}\right)$ and $\mathcal{W}\left(l_{0}+l\right)$ denote the bulk energy of the displacements $u_{\Gamma_{0}}$ and $u_{\Gamma_{l}}$ associated to the boundary datum $\psi$ and the cracks $\Gamma_{0}$ and $\Gamma_{l}$, respectively, and $k$ represents the toughness of the material. Moreover, during a quasistatic crack evolution, if $\Gamma_{0}$ propagates along the $\Gamma_{l}$, then (5.2) holds with equality.

Let us prove that the rate of energy release that appears in the left-hand side of (5.2) is zero in the case in which $\Gamma_{0}=\emptyset$ and the elastic solution $u$ relative to the boundary displacement $\psi$ has at most uniformly weak singularities. This means that the elastic configuration is always in equilibrium according to Griffith's theory, and moreover that a quasistatic crack evolution which begins in the elastic configuration remains at all subsequent times in the elastic regime, that is, Griffith's theory cannot explain crack initiation.

To this aim for every $l>0$, let us set

$$
\mathcal{W}(l):=\inf \left\{\int_{\Omega} f\left(x, \nabla u_{\Gamma}\right) \mathrm{d} x: \Gamma \in \mathcal{K}_{m}(\Omega), \mathcal{H}^{1}(\Gamma) \leqq l\right\},
$$

where $u_{\Gamma} \in W^{1, p}(\Omega \backslash \Gamma)$ denotes the displacement associated to $\Gamma$ and the boundary datum $\psi$. Notice that we clearly have $\mathcal{W}(0)=\int_{\Omega} f(x, \nabla u) \mathrm{d} x$, where $u$ is the elastic displacement relative to $\psi$. The following proposition holds.

Proposition 3. Let us assume that the hypotheses of Theorem 3 are fulfilled. Then we have

$$
\lim _{l \rightarrow 0^{+}} \frac{\mathcal{W}(0)-\mathcal{W}(l)}{l}=0
$$

Proof. For every $\tilde{k}>0$, by Theorem 3 we have that for $l$ small enough and $\Gamma \in \mathcal{K}_{m}(\bar{\Omega})$ with $\mathcal{H}^{1}(\Gamma) \leqq l$

$$
\int_{\Omega} f(x, \nabla u) \mathrm{d} x \leqq \int_{\Omega} f\left(x, \nabla u_{\Gamma}\right)+\tilde{k} \mathcal{H}^{1}(\Gamma) .
$$

We deduce that

$$
\limsup _{l \rightarrow 0^{+}} \frac{\mathcal{W}(0)-\mathcal{W}(l)}{l} \leqq \tilde{k}
$$

Since $\tilde{k}$ is arbitrary, and since $\mathcal{W}(l) \leqq \mathcal{W}(0)$, we conclude that the result holds.

Remark 8. (The case of strong singularities) If the elastic solution $u$ has a strong singularity at $x \in \Omega$, then by Remark 4 we have that

$$
\lim _{l \rightarrow 0^{+}} \frac{\mathcal{W}(0)-\mathcal{W}(l)}{l}=+\infty
$$

so that the elastic configuration is not in equilibrium in the framework of Griffith's theory. 


\subsection{Crack initiation in variational theories of crack propagation}

As explained at the beginning of the section, we now consider irreversible quasistatic crack evolutions governed by a static equilibrium condition and an energy balance. More precisely if $\psi(t)$ is a time-dependent boundary displacement, and $u(t), \Gamma(t)$ are the displacement and the crack at time $t$ relative to $\psi(t)$, we assume that the pair $(u(t), \Gamma(t))$ satisfies the following properties:

(a) Irreversibility: $\Gamma(t)$ is increasing in time, that is, $\Gamma\left(t_{1}\right) \subseteq \Gamma\left(t_{2}\right)$ for all $0 \leqq$ $t_{1} \leqq t_{2} \leqq T$

(b) Static equilibrium: if $t>0, \mathcal{E}(u(t), \Gamma(t)) \leqq \mathcal{E}(u, H)$ for all cracks $H$ such that $\cup_{s<t} \Gamma(s) \subseteq H$ and all displacements $v: \Omega \backslash H \rightarrow \mathbb{R}$ with $v=\psi(t)$ on $\partial_{D} \Omega \backslash H$

(c) Energy balance: the total energy $\mathcal{E}(u(t), \Gamma(t))$ is absolutely continuous in time, and it satisfies

$$
\mathcal{E}(u(t), \Gamma(t))=\mathcal{E}(u(0), \Gamma(0))+\int_{0}^{t} \int_{\Omega} \partial f(x, \nabla u(\tau)) \nabla \dot{\psi}(\tau) \mathrm{d} x \mathrm{~d} \tau .
$$

Condition (a) stands for the irreversibility of the evolution: the crack can only increase in time, that is, no healing processes are admitted. Condition $(b)$ asserts that the pair $(u(t), \Gamma(t))$ is a unilateral minimizer of the total energy, that is, it is a minimum among all configuration with larger cracks. In particular $u(t)$ is the elastic deformation relative to the boundary datum $\psi(t)$ in the domain $\Omega \backslash \Gamma(t)$, that is, $u(t)$ satisfies equation (2.5) with $\Gamma=\Gamma(t)$ and $\psi=\psi(t)$. Finally, notice that under suitable regularity assumptions on $u(t)$ and $\Gamma(t)$, condition $(c)$ can be rewritten as

$$
\begin{gathered}
\int_{\Omega} f(x, \nabla u(t)) \mathrm{d} x-\int_{\Omega} f(x, \nabla u(s)) \mathrm{d} x+k \mathcal{H}^{1}(K(t) \backslash K(s)) \\
=\int_{s}^{t} \int_{\partial_{D} \Omega \backslash K(\tau)} \frac{\partial}{\partial n} f(x, \nabla u(\tau)) \cdot \dot{\psi}(\tau) \mathrm{d} \mathcal{H}^{1}(x) \mathrm{d} \tau .
\end{gathered}
$$

Therefore the energy balance condition states that the sum of the variation of the bulk energy and of the dissipation due to the creation of a new crack is equal to the work inserted in the system by the boundary datum $\psi$. We refer the reader to the paper by FRANCFORT and MARIGO [20] for further details on crack evolutions satisfying conditions $(a),(b)$, and $(c)$ (see also MiELKE [27] for a connection with the theory of rate-independent processes).

In order to treat the problem of crack initiation, we consider as in [20] the case in which $f$ is $p$-homogeneous in the gradient, that is, for all $x \in \Omega, \xi \in \mathbb{R}^{2}$ and $t>0$

$$
f(x, t \xi)=t^{p} f(x, \xi)
$$

We consider a time-dependent boundary displacement of the form $t \rightarrow t \psi$, where $t \in[0, T]$ and $\psi \in W^{1, p}(\Omega) \cap L^{\infty}(\Omega)$ is a given function. We refer the 
reader to the paper by DAL MASO and TOADER [14] for the existence of a quasistatic crack evolution $\{t \rightarrow(u(t), \Gamma(t))\}$ with

$$
\Gamma(t) \in \mathcal{K}_{m}(\bar{\Omega}) \text { and } u(t) \in W^{1, p}(\Omega \backslash \Gamma(t))
$$

for every $t \in[0, T]$ satisfying $(a),(b)$, and $(c)$.

Since we are dealing with a crack initiation problem, and since $\psi(0)=0$, we assume that $(u(0), \Gamma(0))=(0, \varnothing)$. Notice that, if $v$ denotes the elastic displacement associated to the boundary datum $\psi$, then $t v$ is the elastic displacement associated to $t \psi$. Then from the static equilibrium condition, comparing $(u(t), \Gamma(t))$ with $(t v, \Gamma(t))$, we have that for all $t \in] 0, T]$

$$
\int_{\Omega} f(x, \nabla u(t)) \mathrm{d} x \leqq t^{p} \int_{\Omega} f(x, \nabla v) \mathrm{d} x .
$$

Finally, since we can replace $t \psi$ by $t v$ in the energy balance condition, we can write

$$
\int_{\Omega} f(x, \nabla u(t)) \mathrm{d} x+k \mathcal{H}^{1}(\Gamma(t))=\int_{0}^{t} \int_{\Omega} \partial f(x, \nabla u(\tau)) \cdot \nabla v \mathrm{~d} x \mathrm{~d} \tau .
$$

This implies that

$$
\int_{\Omega} f(x, \nabla u(t)) \mathrm{d} x+k \mathcal{H}^{1}(\Gamma(t)) \leqq t^{p} \int_{\Omega} f(x, \nabla v) \mathrm{d} x .
$$

In fact, from the inequality

$$
z w \leqq f(x, z)+f^{*}(x, w),
$$

taking into account the $p$-homogeneity of $f$, we can write for $a, b>0$

$$
(z / a)(w / b) \leqq\left(1 / a^{p}\right) f(x, z)+\left(1 / b^{q}\right) f^{*}(x, w)
$$

where $q=p^{\prime}:=p /(p-1)$. For $z, w: \Omega \rightarrow \mathbb{R}$ measurable functions on $\Omega$, integrating over $\Omega$, and setting

$$
a^{p}=p \int_{\Omega} f(x, z) \mathrm{d} x \text { and } b^{q}=q \int_{\Omega} f(x, w) \mathrm{d} x,
$$

we obtain the following Hölder-type inequality

$$
\int_{\Omega} z w \leqq p^{1 / p} q^{1 / q}\left(\int_{\Omega} f(x, z) \mathrm{d} x\right)^{1 / p}\left(\int_{\Omega} f^{*}(x, w) \mathrm{d} x\right)^{1 / q} .
$$

Taking $z=\nabla v$ and $w=\partial f(x, \nabla u(\tau))$ we get [using $f^{*}(\partial f(z))+f(z)=\partial f(z) \cdot z$ $=p f(z)]$

$$
\begin{aligned}
& \int_{\Omega} \partial f(x, \nabla u(\tau)) \nabla v \mathrm{~d} x \\
& \quad \leqq p^{1 / p} q^{1 / q}\left(\int_{\Omega} f(x, \nabla v) \mathrm{d} x\right)^{\frac{1}{p}}\left(\int_{\Omega} f^{*}(x, \partial f(x, \nabla u(\tau))) \mathrm{d} x\right)^{\frac{1}{q}} \\
& \quad=p^{1 / p} q^{1 / q}\left(\int_{\Omega} f(x, \nabla v) \mathrm{d} x\right)^{\frac{1}{p}}(p-1)^{\frac{1}{q}}\left(\int_{\Omega} f(x, \nabla u(\tau)) \mathrm{d} x\right)^{\frac{1}{q}} .
\end{aligned}
$$


In view of (5.4) we have

$$
\int_{\Omega} \partial f(x, \nabla u(\tau)) \nabla v \mathrm{~d} x \leqq \tau^{\frac{p}{q}} p^{1 / p} q^{1 / q}(p-1)^{\frac{1}{q}} \int_{\Omega} f(x, \nabla v) \mathrm{d} x .
$$

Integrating from 0 to $t$ we obtain

$$
\int_{0}^{t} \int_{\Omega} \partial f(x, \nabla u(\tau)) \nabla v \mathrm{~d} x \leqq p^{1 / p} q^{1 / q} p^{-1}(p-1)^{\frac{1}{q}} t^{p} \int_{\Omega} f(x, \nabla v) \mathrm{d} x .
$$

Since

$$
p^{1 / p} q^{1 / q} p^{-1}(p-1)^{\frac{1}{q}}=1,
$$

by (5.5) we conclude that (5.6) holds.

Notice that we can rescale (5.6) obtaining for $t$ small

$$
\int_{\Omega} f(x, \nabla v(t)) \mathrm{d} x+\mathcal{H}^{1}(\Gamma(t)) \leqq \int_{\Omega} f(x, \nabla v) \mathrm{d} x,
$$

where $v(t):=\frac{1}{t} u(t)$ is the displacement associated to $\Gamma(t)$ and $\psi$.

As noticed by FrAnCFORT and MARIGo in [20], if $T$ is large enough, a crack will appear during the evolution, that is, $\Gamma(s) \neq \varnothing$ for some $s \in] 0, T[$. In fact if $T$ is such that

$$
\mathcal{H}^{1}\left(\partial_{D} \Omega\right)<T^{p} \int_{\Omega} f(x, \nabla v) \mathrm{d} x
$$

then we get that creating a crack along $\partial_{D} \Omega$ is more convenient that deforming $\Omega$ elastically. We are now in a position to state the first crack initiation result.

Theorem 4. Let us assume that T satisfies (5.9), and let us suppose that the elastic displacement $v$ associated to the boundary datum $\psi$ has at most uniformly weak singularities in $\Omega$, that is, it satisfies (3.3). Then the crack initiation is brutal, that is, there exists a positive time $\left.\left.t_{i} \in\right] 0, T\right]$ such that $\Gamma(t)=\emptyset$ for every $t \leqq t_{i}$, and $\mathcal{H}^{1}(\Gamma(t))>l^{*}$ for all $\left.\left.t \in\right] t_{i}, T\right]$ for some $l^{*}>0$ depending on $\Omega, f, k, m$, and $\psi$.

Proof. Notice that by (5.6) we have $\mathcal{H}^{1}(\Gamma(t)) \rightarrow 0$ as $t \rightarrow 0$. By Theorem 3, we get that for $t$ small the elastic solution $v$ is energetically convenient with respect to $(v(t), \Gamma(t))$, where $v(t)$ is the displacement associated to $\Gamma(t)$ and $\psi$. But this is against (5.8) unless $\mathcal{H}^{1}(\Gamma(t))=0$. In view of (5.9) we deduce that there exists $\left.t_{i} \in\right] 0, T$ [ such that $\Gamma(t)=\varnothing$ for every $t \leqq t_{i}$ and $\mathcal{H}^{1}(\Gamma(t))>0$ for all $\left.\left.t \in\right] t_{i}, T\right]$.

In order to prove that $\mathcal{H}^{1}(\Gamma(t))>l^{*}$ for all $\left.\left.t \in\right] t_{i}, T\right]$ and for some $l^{*}>0$, notice that by (5.6) we deduce that

$$
\int_{\Omega} f(x, \nabla v(t)) \mathrm{d} x+k t^{-p} \mathcal{H}^{1}(\Gamma(t)) \leqq \int_{\Omega} f(x, \nabla v) \mathrm{d} x .
$$

Then by Theorem 3 we deduce that

$$
\liminf _{t \searrow t_{i}} \mathcal{H}^{1}(\Gamma(t)) \geqq l^{*}
$$

for some positive constant $l^{*}$ depending only on $\Omega, f, k t_{i}^{-p}, m$, and $\psi$. The proof is thus concluded. 
Remark 9. Note that Theorem 4 holds true whenever the elastic solution $v$ is a local minimum for the total energy. Therefore, in view of the results of Section 4 , Theorem 4 can be extended to the setting of planar elasticity. Moreover we will prove (see Theorem 6) that if $v$ is $C^{1}$, and the associated stress is continuous, then $v$ is a local minimizer in the class of $S B V$ displacements. In view of this result, we conclude that crack initiation is always brutal whenever the elastic solution has no singularities at all, without assuming the cracks to be closed, or with a finite number of connected components.

Let us now study the crack initiation in the case in which the elastic displacement $u$ associated to the boundary datum $\psi$ has strong singularities. We recall that a point $x \in \bar{\Omega}$ is a point of strong singularity for $v$ if

$$
\limsup _{r \rightarrow 0} \frac{1}{r} \int_{B_{r}(x) \cap \bar{\Omega}}|\nabla v|^{p} \mathrm{~d} x=+\infty .
$$

It is well expected that during a loading process, a crack will appear at a point of strong singularity. The following theorem establishes this fact for a general quasistatic crack evolution satisfying properties $(a),(b)$, and $(c)$. The result, stated in the setting of antiplane elasticity, can be easily generalized (see Remark 9) to the case of planar elasticity.

Theorem 5. Let us suppose that the elastic displacement $v$ associated to the boundary datum $\psi$ has a strong singularity at $x \in \Omega$, and that $v$ has at most uniformly weak singularities in $\Omega \backslash B_{r}(x)$ for every $r>0$. Then we have that $\mathcal{H}^{1}(\Gamma(t))>0$ for all $t \in] 0, T]$, and the crack starts at the point $\{x\}$, that is,

$$
x \in \bigcap_{t>0} \Gamma(t) .
$$

Moreover the crack departs with zero speed, that is,

$$
\lim _{t \rightarrow 0} \frac{\mathcal{H}^{1}(\Gamma(t))}{t}=0 .
$$

Proof. In view of Remark 4, and since $x$ is a point of strong singularity for $v$, we have that for a positive time $t>0$ the elastic displacement $t v$ relative to the boundary datum $t \psi$ cannot satisfy condition $(b)$. As a consequence, we deduce that $\mathcal{H}^{1}(\Gamma(t))>0$ for all $\left.\left.t \in\right] 0, T\right]$.

Let us come to the properties of $\Gamma$ at time $t=0$. By (5.6) we have

$$
\lim _{t \rightarrow 0} \mathcal{H}^{1}(\Gamma(t))=0
$$

so that

$$
\bigcap_{t>0} \Gamma(t)=\left\{y_{1}, \ldots, y_{h}\right\}
$$

with $h \leqq m$. Let us suppose by contradiction that $y_{i} \neq x$ for all $i=1, \ldots, h$. Then there exists $r>0$ such that for $t$ small enough we have $\Gamma(t) \subseteq \bar{\Omega} \backslash B_{r}(x)$. 
Since $v$ has at most uniformly weak singularities in $A:=\Omega \backslash \bar{B}_{r}(x)$, and in view of Proposition 1, we have that inequality (5.8) implies that $\mathcal{H}^{1}(\Gamma(t))=0$ for $t$ small, which is a contradiction.

Finally, in order to prove (5.10), we rescale (5.6), obtaining

$$
\int_{\Omega} f(x, \nabla v(t)) \mathrm{d} x+\frac{k}{t^{p}} \mathcal{H}^{1}(\Gamma(t)) \leqq \int_{\Omega} f(x, \nabla v) \mathrm{d} x,
$$

where $v(t)$ is the displacement associated to $\Gamma(t)$ and $\psi$. We deduce that $\mathcal{H}^{1}(\Gamma(t)) \leqq$ $C t^{p}$ for some constant $C$, and so (5.10) easily follows.

\section{Appendix A: The two-dimensional $S B V$ case}

The aim of this appendix is to prove a minimality result along the lines of Theorem 3 which does not require an a priori bound on the number of the connected components of the admissible cracks. Small cracks are still not energetically convenient if the gradient of the elastic solution of problem (2.4) and the related stress are continuous in $\bar{\Omega}$. This condition excludes however that the elastic configuration presents (weak) singularities.

In order to make the mathematical setting of this section precise, we need to recall some facts about rectifiable sets and the functional space $S B V$ of special functions with bounded variation. We refer the reader to [4] for a complete treatment of these subjects.

A set $\Gamma \subseteq \mathbb{R}^{N}$ is rectifiable if there exists $N_{0} \subseteq \Gamma$ with $\mathcal{H}^{N-1}\left(N_{0}\right)=0$, and a sequence $\left(\bar{M}_{i}\right)_{i \in \mathbb{N}}$ of $C^{1}$-submanifolds of $\mathbb{R}^{N}$ such that

$$
\Gamma \backslash N_{0} \subseteq \bigcup_{i \in \mathbb{N}} M_{i}
$$

For every $x \in \Gamma \cap M_{i}$, we define the normal to $\Gamma$ at $x$ as $n_{M_{i}}(x)$. It turns out that the normal is well defined (up to the sign) for $\mathcal{H}^{N-1}$-almost every $x \in \Gamma$.

Let $U \subseteq \mathbb{R}^{N}$ be an open bounded set with Lipschitz boundary. $S B V(U)$ is the set of functions $u \in L^{1}(U)$ such that the distributional derivative $D u$ is a Radon measure which, for every open set $A \subseteq U$, can be represented as

$$
D u(A)=\int_{A} \nabla u \mathrm{~d} x+\int_{A \cap S(u)}[u](x) v \mathrm{~d} \mathcal{H}^{N-1}(x),
$$

where $\nabla u$ is the approximate differential of $u, S(u)$ is the set of jump of $u$ (which is a rectifiable set), $v(x)$ is the normal to $S(u)$ at $x$, and $[u](x)$ is the jump of $u$ at $x$.

For every $p \in] 1,+\infty[$ we set

$$
S B V^{p}(U):=\left\{u \in S B V(U): \nabla u \in L^{p}\left(U, \mathbb{R}^{N}\right), \mathcal{H}^{N-1}(S(u))<+\infty\right\} .
$$

If $u \in S B V(U)$, then $u$ admits a trace $\gamma(u)$ on $\partial U$ which is characterized by the relation (see [4, Theorem 3.87])

$$
\lim _{r \rightarrow 0} r^{-N} \int_{\Omega \cap B_{r}(x)}|u(y)-\gamma(u)(x)| \mathrm{d} y=0 \text { for } \mathcal{H}^{N-1} \text {-almost every } x \in \partial U .
$$


We will denote the trace $\gamma(u)$ on $\partial U$ again by $u$. If $\Gamma \subseteq U$ is rectifiable and oriented by a normal vector field $n$, then we can define the traces $\gamma_{\Gamma}^{+}(u)$ and $\gamma_{\Gamma}^{-}(u)$ of $u \in S B V(U)$ on $\Gamma$ (see [4, Theorem 3.77]) which are characterized by the relations

$$
\lim _{r \rightarrow 0} r^{-N} \int_{\Omega \cap B_{r}^{ \pm}(x)}\left|u(y)-\gamma_{\Gamma}^{ \pm}(u)(x)\right| \mathrm{d} y=0 \text { for } \mathcal{H}^{N-1} \text {-almost every } x \in \Gamma,
$$

where $B_{r}^{ \pm}(x):=\left\{y \in B_{r}(x):(y-x) \cdot n \gtrless 0\right\}$. It turns out that the jump $[u](x)$ for $\mathcal{H}^{N-1}$-almost every $x \in S(u)$ is given by the difference of the traces of $u$ at $x$ on both sides of $S(u)$.

A set $E \subseteq U$ has finite perimeter in $U$ if the characteristic function $1_{E}$ belongs to $S B V(U)$. We denote by $\partial^{*} E$ the set of jumps of $1_{E} \cdot \partial^{*} E$ is usually referred to as the reduced boundary of $E$ in $U$.

Let us now come to our problem of local minimality. Let $\Omega \subseteq \mathbb{R}^{2}$ be open, connected, and with Lipschitz boundary. Let $\partial_{D} \Omega \subseteq \partial \Omega$ be open in the relative topology, and let $\partial_{N} \Omega:=\partial \Omega \backslash \partial_{D} \Omega$. Let $f(x, \xi)$ be a Carathéodory function satisfying (2.1), (2.2), and (2.3). Let us moreover assume that the boundary displacement on $\partial_{D} \Omega$ is given by the trace of a continuous function $\psi \in C^{0}(\bar{\Omega}) \cap W^{1, p}(\Omega)$. We denote by $u$ the elastic solution relative to $\psi$, namely the solution to the problem

$$
\min \left\{\int_{\Omega} f(x, \nabla u) \mathrm{d} x: u \in W^{1, p}(\Omega), u=\psi \text { on } \partial_{D} \Omega\right\} .
$$

The class of admissible cracks we consider is

$$
\mathcal{R}(\bar{\Omega}):=\left\{\Gamma \subseteq \bar{\Omega}: \Gamma \text { is rectifiable and } \mathcal{H}^{1}(\Gamma)<+\infty\right\}
$$

Let us come to the class of admissible displacements relative to a crack $\Gamma$ and to the boundary displacement $\psi$. Since $\Gamma$ is not supposed to be closed, the Sobolev space $W^{1, p}(\Omega \backslash \Gamma)$ is not well defined. So we consider as class of admissible displacements the functions $u \in S B V^{p}(\Omega)$ such that $S^{\psi}(u) \subseteq \Gamma$, where

$$
S^{\psi}(u):=S(u) \cup\left\{x \in \partial_{D} \Omega: u(x) \neq \psi(x)\right\},
$$

and the inequality on $\partial_{D} \Omega$ is intended for the traces. Notice that if $\Gamma$ is closed then $u \in W^{1, p}(\Omega \backslash \Gamma)$, and $u=\psi$ on $\partial_{D} \Omega \backslash \Gamma$.

The displacement $u_{\Gamma} \in S B V^{p}(\Omega) \cap L^{\infty}(\Omega)$ associated to $\Gamma$ and $\psi$ is a solution of the minimum problem

$$
\min \left\{\int_{\Omega} f(x, \nabla u) \mathrm{d} x: u \in S B V^{p}(\Omega), S^{\psi}(u) \subseteq \Gamma\right\}
$$

The proof of the existence of $u_{\Gamma}$ is standard: it relies on Ambrosio's compactness and lower semicontinuity Theorem [2], together with a truncation argument.

The main result of the section is the following. 
Theorem 6. Let $u$ be the elastic displacement relative to $\psi \in W^{1, p}(\Omega) \cap C^{0}(\bar{\Omega})$, and let us assume that $u$ satisfies

$$
\nabla u \in C^{0}\left(\bar{\Omega} ; \mathbb{R}^{2}\right) \text { and } \sigma:=\partial_{\xi} f(x, \nabla u) \in C^{0}\left(\bar{\Omega} ; \mathbb{R}^{2}\right) .
$$

Then there exists a critical length $l^{*}>0$ depending on $\Omega, f, k$, and $\psi$ such that for all $\Gamma \in \mathcal{R}(\bar{\Omega})$ with $\mathcal{H}^{1}(\Gamma)<l^{*}$ we have

$$
\int_{\Omega} f(x, \nabla u) \mathrm{d} x<\int_{\Omega} f\left(x, \nabla u_{\Gamma}\right) \mathrm{d} x+k \mathcal{H}^{1}(\Gamma) .
$$

In order to prove Theorem 6 we need the following lemma.

Lemma 3. For every $\Gamma \in \mathcal{R}(\bar{\Omega})$ we have

$$
\int_{\Omega}\left[f(x, \nabla u)-f\left(x, \nabla u_{\Gamma}\right)\right] \mathrm{d} x \leqq \int_{\Gamma} \sigma \cdot n\left(u_{\Gamma}^{+}-u_{\Gamma}^{-}\right) \mathrm{d} \mathcal{H}^{1},
$$

where $\sigma$ is the stress of the elastic displacement $u$ defined in (A.3), $u_{\Gamma}$ is a minimum of (A.2), and $u_{\Gamma}^{ \pm}$are the traces of $u_{\Gamma}$ on $\Gamma$ (if $\Gamma$ touches $\partial_{D} \Omega$, we set $u_{\Gamma}^{+}(x)=\psi(x)$ and $u_{\Gamma}^{-}(x)=\gamma(u)(x), \gamma(u)$ being the trace of $u$ on $\partial \Omega$, while if $\Gamma$ touches $\partial_{N} \Omega$ we set $\left.u_{\Gamma}^{ \pm}(x)=\psi(x)\right)$.

Proof. By the convexity of $f$ we have

$$
\begin{aligned}
\int_{\Omega}\left[f(x, \nabla u)-f\left(x, \nabla u_{\Gamma}\right)\right] \mathrm{d} x & \leqq \int_{\Omega} \partial f(x, \nabla u)\left(\nabla u-\nabla u_{\Gamma}\right) \mathrm{d} x \\
& =\int_{\Omega} \sigma\left(\nabla u-\nabla u_{\Gamma}\right) \mathrm{d} x .
\end{aligned}
$$

We can assume that $\psi$ is defined on $\mathbb{R}^{2}$, that is, $\psi \in W^{1, p}\left(\mathbb{R}^{2}\right) \cap C^{0}\left(\mathbb{R}^{2}\right)$. Let $B$ be a ball centered at 0 such that $\bar{\Omega} \subseteq B$. Let us set $\Omega^{\prime}:=B \backslash \partial_{N} \Omega$. We can extend $u$ and $u_{\Gamma}$ to $\Omega^{\prime}$ setting $u=u_{\Gamma}=\psi$ on $B \backslash \bar{\Omega}$. Let us consider $u_{n} \in C^{1}\left(\Omega^{\prime}\right)$ with $u_{n}=\psi$ on $B \backslash \bar{\Omega}$ and such that

$$
D u_{n} \rightarrow D u_{\Gamma} \quad \text { strictly in the sense of measures, }
$$

that is (see [4, Theorem 3.9])

$$
\lim _{n \rightarrow+\infty} \int_{\Omega^{\prime}} \varphi \mathrm{d} D u_{n}=\int_{\Omega^{\prime}} \varphi \mathrm{d} D u_{\Gamma} \quad \text { for all } \varphi \in C^{0}\left(\Omega^{\prime}\right) \cap L^{\infty}\left(\Omega^{\prime}\right) .
$$

Since $u$ is a minimum for problem (A.2) with $\Gamma=\emptyset$, and $u_{n}=\psi$ on $\partial_{D} \Omega$, then we have that $u-u_{n}$ is an admissible variation for $u$ so that for all $n \in \mathbb{N}$

$$
0=\int_{\Omega} \sigma\left(\nabla u-\nabla u_{n}\right) \mathrm{d} x=\int_{\Omega} \sigma \mathrm{d} D\left(u-u_{n}\right) .
$$

Since by assumption $\sigma \in C^{0}(\bar{\Omega})$, we can extend $\sigma$ to $\Omega^{\prime}$ in such a way that $\sigma \in C^{0}\left(\Omega^{\prime}\right) \cap L^{\infty}\left(\Omega^{\prime}\right)$. Notice that since $u=u_{n}=\psi$ on $B \backslash \bar{\Omega}$, from (A.6) we have

$$
\int_{\Omega^{\prime}} \sigma \mathrm{d} D\left(u_{n}-u\right)=0
$$


Then by strict convergence we deduce

$$
\begin{aligned}
0 & =\lim _{n \rightarrow \infty} \int_{\Omega^{\prime}} \sigma \mathrm{d} D\left(u-u_{n}\right)=\int_{\Omega^{\prime}} \sigma \nabla u \mathrm{~d} x-\int_{\Omega^{\prime}} \sigma \mathrm{d} D u_{\Gamma} \\
& =\int_{\Omega^{\prime}} \sigma\left(\nabla u-\nabla u_{\Gamma}\right) \mathrm{d} x-\int_{S\left(u_{\Gamma}\right)} \sigma \cdot n\left(u_{\Gamma}^{+}-u_{\Gamma}^{-}\right) d \mathcal{H}^{1} .
\end{aligned}
$$

Since $S\left(u_{\Gamma}\right) \subseteq \Gamma, u_{\Gamma}^{+}=u_{\Gamma}^{-}$on $\Gamma \backslash S\left(u_{\Gamma}\right)$, and $\nabla u=\nabla u_{\Gamma}=\nabla \psi$ on $B \backslash \bar{\Omega}$, by (A.7) we deduce

$$
\int_{\Gamma} \sigma \cdot n\left(u_{\Gamma}^{+}-u_{\Gamma}^{-}\right) \mathrm{d} \mathcal{H}^{1}=\int_{\Omega^{\prime}} \sigma\left(\nabla u-\nabla u_{\Gamma}\right) \mathrm{d} x=\int_{\Omega} \sigma\left(\nabla u-\nabla u_{\Gamma}\right) \mathrm{d} x,
$$

so that, in view of (A.5), we have that (A.4) follows.

We are now in a position to prove the minimality result in the $S B V$ context.

Proof (Proof of Theorem 6). Let us consider $l>0$, and let $\Gamma \in \mathcal{R}(\bar{\Omega})$ be a minimum for the functional

$$
\mathcal{F}(\Gamma):=\int_{\Omega} f\left(x, \nabla u_{\Gamma}\right) \mathrm{d} x+k \mathcal{H}^{1}(\Gamma)
$$

among the cracks $\Gamma \in \mathcal{R}(\bar{\Omega})$ such that $\mathcal{H}^{1}(\Gamma) \leqq l$. The existence of such a $\Gamma$ follows by taking a minimum $\tilde{v}$ of the functional

$$
F(v):=\int_{\Omega} f(x, \nabla v) \mathrm{d} x+k \mathcal{H}^{1}\left(S^{\psi}(v)\right)
$$

among all $v \in S B V^{p}(\Omega)$ with $\mathcal{H}^{1}\left(S^{\psi}(v)\right) \leqq l$, and choosing $\Gamma:=S^{\psi}(\tilde{v})$. As a consequence, we can assume that $\Gamma \cap \partial_{N} \Omega=\emptyset$, and moreover that for every $x \in \Gamma$ we have $\left|\left[u_{\Gamma}\right](x)\right|>0$ [on $\partial_{D} \Omega$ we mean $|\psi(x)-\gamma(u)(x)|$, with $\gamma(u)$ the trace of $u$ on $\partial \Omega$ ]. Theorem 6 will be proved if we show that $\Gamma=\emptyset$ for $l$ small enough.

The main idea to prove that $\Gamma=\emptyset$ for $l$ small enough is to look at the the quantity $\left|\left[u_{\Gamma}\right]\right|$, to prove that it is infinitesimal as $l \rightarrow 0$, and to apply (A.4). There are some problems connected to this strategy. Recall that, while the strain $\nabla u_{\Gamma}$ is uniquely determined, the displacement $u_{\Gamma}$ is not, because, at least if $\Gamma$ is closed, $u_{\Gamma}$ can be any constant on the connected components of $\Omega \backslash \Gamma$ which do not touch $\partial_{D} \Omega$. This is also the case, in a suitable weak sense, when $\Gamma$ is only rectifiable. Consider indeed $E \subseteq \Omega$ with finite perimeter in $\mathbb{R}^{2}$, such that $\partial^{*} E \subseteq \Gamma \cup \partial_{N} \Omega$, where $\partial^{*} E$ denotes the reduced boundary of $E$ with respect to $\mathbb{R}^{2}$. Notice that we can assume that $u_{\Gamma}$ is equal to a constant $c$ on $E$. In fact we have that

$$
\tilde{u}_{\Gamma}:= \begin{cases}u_{\Gamma} & \text { in } \Omega \backslash E \\ c & \text { in } E\end{cases}
$$

belongs to $S B V(\Omega)$, and it is an admissible displacement for $\Gamma$ and $\psi$ (see [4, Theorem 3.84]) with

$$
\int_{\Omega} f\left(x, \nabla \tilde{u}_{\Gamma}\right) \mathrm{d} x \leqq \int_{\Omega} f\left(x, \nabla u_{\Gamma}\right) \mathrm{d} x .
$$


We conclude that $\tilde{u}_{\Gamma}$ is a minimum energy displacement relative to $\Gamma$ and $\psi$. Coming back to $\left|\left[u_{\Gamma}\right]\right|$, we see that this quantity is not well defined unless we fix a representative of $u_{\Gamma}$. Moreover, having fixed such a representative, we have that we can estimate at most the oscillation of $\left[u_{\Gamma}\right]$ on $\partial^{*} E$, and not prove that $\left|\left[u_{\Gamma}\right]\right|$ converges to zero as $l \rightarrow 0$. So in what follows, we split $\Gamma$ in two pieces $\Gamma \backslash \Gamma^{*}$ and $\Gamma^{*}$, where $\Gamma^{*}$ is related to the sets $E$ on which $u_{\Gamma}$ is constant [so that only an estimate for the oscillation holds, see (A.15)], while on the rest the quantity $\left[u_{\Gamma}\right]$ tends to zero as $l \rightarrow 0$ [see (A.14)].

Let $E \subseteq \Omega$ be the set with finite perimeter in $\mathbb{R}^{2}$, maximal with respect to inclusion, such that $\partial^{*} E \subseteq \Gamma \cup \partial_{N} \Omega$. As we have seen, we can assume that

$$
u_{\Gamma}=0 \text { on } E \text {. }
$$

Notice that, in view of (A.8), we may assume also that if $x \in \Gamma \backslash \partial^{*} E$, then $x$ has not density 1 for $E$, that is,

$$
\liminf _{r \rightarrow 0^{+}} \frac{|E \cap B(x, r)|}{|B(x, r)|}<1 .
$$

Otherwise, we would get $\left[u_{\Gamma}\right](x)=0$.

Let us divide $E$ in the union of its indecomposable components according to [5, Theorem 1], that is, let $\left(E_{i}\right)_{i \in \mathbb{N}}$ be a family of sets with finite perimeter in $\mathbb{R}^{2}$ such that $E=\bigcup_{i \in \mathbb{N}} E_{i}, \mathcal{H}^{1}\left(\partial^{*} E\right)=\sum_{i \in \mathbb{N}} \mathcal{H}^{1}\left(\partial^{*} E_{i}\right),\left|E_{h} \cap E_{k}\right|=0$, $\mathcal{H}^{1}\left(\partial^{*} E_{h} \cap \partial^{*} E_{k}\right)=0$ for every $h \neq k$, and such that for every $k \in \mathbb{N}$ the set $E_{k}$ cannot be written as $E_{k}=E_{k}^{1} \cup E_{k}^{2}$ with $\left|E_{k}^{1} \cap E_{k}^{2}\right|=0$ and $\mathcal{H}^{1}\left(\partial^{*} E_{k}\right)=$ $\mathcal{H}^{1}\left(\partial^{*} E_{k}^{1}\right)+\mathcal{H}^{1}\left(\partial^{*} E_{k}^{2}\right)$. Let us set

$$
\Gamma^{*}:=\partial^{*} E \backslash \partial_{N} \Omega=\left(\bigcup_{i=0}^{\infty} \partial^{*} E_{i}\right) \backslash \partial_{N} \Omega,
$$

and let us assume that $n$ denotes the outward normal to $E_{i}$. Since the stress $\sigma$ is a divergence-free vector field, in view of the generalized Gauss-Green formula for sets with finite perimeter (see [4, Theorem 3.36]) we have for all $i \in \mathbb{N}$

$$
\int_{\partial^{*} E_{i}} \sigma \cdot n \mathrm{~d} \mathcal{H}^{1}=\int_{E_{i}} \operatorname{div} \sigma \mathrm{d} x=0 .
$$

In order to prove our minimality result, by contradiction let us assume that there exists $l_{h} \rightarrow 0$ and $\Gamma_{h}$ with $\mathcal{H}^{1}\left(\Gamma_{h}\right) \leqq l_{h}$, such that setting $u_{h}:=u_{\Gamma_{h}}$ we have

$$
\int_{\Omega} f\left(x, \nabla u_{h}\right) \mathrm{d} x+k \mathcal{H}^{1}\left(\Gamma_{h}\right)<\int_{\Omega} f(x, \nabla u) \mathrm{d} x .
$$

By Ambrosio's lower semicontinuity Theorem [2] and by (A.12), we deduce that for every open set $A \subseteq \Omega$

$$
\lim _{h \rightarrow+\infty} \int_{A} f\left(x, \nabla u_{h}\right) \mathrm{d} x=\int_{A} f(x, \nabla u) \mathrm{d} x .
$$


Let $E^{h},\left(E_{i}^{h}\right)_{i \in \mathbb{N}}$ and $\Gamma_{h}^{*}$ be the sets associated to $\Gamma_{h}$ described above. We claim that

$$
\left|\left[u_{h}\right]\right|:=\left|u_{h}^{+}-u_{h}^{-}\right| \rightarrow 0 \text { uniformly on } \Gamma_{h} \backslash \Gamma_{h}^{*} \text { as } h \rightarrow+\infty
$$

and

$\operatorname{ess}^{-\sup _{\partial^{*}} E_{i}^{h}}\left(u_{h}^{+}\right)-$ess-inf $_{\partial^{*} E_{i}^{h}}\left(u_{h}^{+}\right) \rightarrow 0$ uniformly in $i$ as $h \rightarrow+\infty$.

If $x_{h} \in \Gamma_{h} \backslash \Gamma_{h}^{*}$ and $x_{h} \in \partial_{D} \Omega$, we intend $\psi\left(x_{h}\right)$ for $u_{h}^{+}\left(x_{h}\right)$, and $\gamma\left(u_{h}\right)(x)$ for $u_{h}^{-}(x)$ [with $\gamma(u)$ the trace of $u$ on $\partial \Omega$ ], while if $x_{h} \in \partial^{*} E_{i}^{h} \cap \partial_{N} \Omega$, we intend $\psi\left(x_{h}\right)=u_{h}^{+}\left(x_{h}\right)$.

In view of (A.14) and (A.15), the proof of the proposition is readily concluded. In fact, given $\varepsilon>0$, and choosing $h$ so large that

$$
\left|\left[u_{h}\right]\right| \leqq \varepsilon \quad \text { on } \Gamma_{h} \backslash \Gamma_{h}^{*}
$$

and for all $i \in \mathbb{N}$

$$
\operatorname{ess-sup}_{\partial^{*}} E_{i}^{h} \backslash \partial_{N} \Omega\left(u_{h}^{+}\right)-\operatorname{ess-inf}_{\partial^{*} E_{i}^{h} \backslash \partial_{N} \Omega}\left(u_{h}^{+}\right) \leqq \varepsilon,
$$

by Lemma 3, in view also of (A.8) and of (A.11), and recalling that $\sigma \cdot n=0$ on $\partial_{N} \Omega$, we have that

$$
\begin{aligned}
\int_{\Omega}[ & \left.f(x, \nabla u)-f\left(x, \nabla u_{h}\right)\right] \mathrm{d} x \\
\leqq & \int_{\Gamma_{h}} \sigma \cdot n\left(u_{h}^{+}-u_{h}^{-}\right) \mathrm{d} \mathcal{H}^{1} \\
= & \int_{\Gamma_{h} \backslash \Gamma_{h}^{*}} \sigma \cdot n\left(u_{h}^{+}-u_{h}^{-}\right) \mathrm{d} \mathcal{H}^{1}+\int_{\Gamma_{h}^{*}} \sigma \cdot n\left(u_{h}^{+}-u_{h}^{-}\right) \mathrm{d} \mathcal{H}^{1} \\
= & \int_{\Gamma_{h} \backslash \Gamma_{h}^{*}} \sigma \cdot n\left(u_{h}^{+}-u_{h}^{-}\right) \mathrm{d} \mathcal{H}^{1}+\sum_{i=0}^{\infty} \int_{\partial^{*} E_{i}^{h} \backslash \partial_{N} \Omega} \sigma \cdot n u_{h}^{+} \mathrm{d} \mathcal{H}^{1} \\
= & \int_{\Gamma_{h} \backslash \Gamma_{h}^{*}} \sigma \cdot n\left(u_{h}^{+}-u_{h}^{-}\right) \mathrm{d} \mathcal{H}^{1}+\sum_{i=0}^{\infty} \int_{\partial^{*} E_{i}^{h}} \sigma \cdot n u_{h}^{+} \mathrm{d} \mathcal{H}^{1} \\
\leqq & \varepsilon\|\sigma\|_{\infty} \mathcal{H}^{1}\left(\Gamma_{h} \backslash \Gamma_{h}^{*}\right) \\
& +\sum_{i=0}^{\infty}\left(\operatorname{ess}^{-i n f} \partial^{*} E_{i}^{h} u_{h}^{+}\right) \times \int_{\partial^{*} E_{i}^{h}} \sigma \cdot n \mathrm{~d} \mathcal{H}^{1}+\varepsilon\|\sigma\|_{\infty} \sum_{i=0}^{\infty} \mathcal{H}^{1}\left(\partial^{*} E_{i}^{h}\right) \\
= & \varepsilon\|\sigma\|_{\infty}\left(\mathcal{H}^{1}\left(\Gamma_{h} \backslash \Gamma_{h}^{*}\right)+\mathcal{H}^{1}\left(\Gamma_{h}^{*} \cup \partial_{N} \Omega\right)\right)=\varepsilon\|\sigma\|_{\infty} \mathcal{H}^{1}\left(\Gamma_{h} \cup \partial_{N} \Omega\right)
\end{aligned}
$$

and this is against (A.12). 
In order to conclude the proof, we have to prove the claims (A.14) and (A.15). Let us consider (A.14), the proof of the other claim being similar. Let us assume that there exists $\delta>0$ and $x_{h} \in \Gamma_{h} \backslash \Gamma_{h}^{*}$ with

$$
\left|\left[u_{h}\right]\left(x_{h}\right)\right| \geqq \delta>0 .
$$

Up to a subsequence we have $x_{h} \rightarrow \bar{x} \in \bar{\Omega}$. Let us assume that $\bar{x} \in \Omega$. For $h$ large enough, and for $r$ small we have $\bar{B}_{r}\left(x_{h}\right) \subseteq \Omega$. Notice that for almost every $r$ such that $\partial B_{r}\left(x_{h}\right) \cap \Gamma_{h}=\emptyset$, we have that $u_{h} \in W^{1, p}\left(\partial B_{r}\left(x_{h}\right)\right)$. Moreover by the maximum principle we have that

$$
\max _{\partial B_{r}\left(x_{h}\right)} u_{h}-\min _{\partial B_{r}\left(x_{h}\right)} u_{h}=M_{h}-m_{h}>\delta .
$$

In fact otherwise, we can consider $\tilde{u}_{h}$ defined as

$$
\tilde{u}_{h}:= \begin{cases}u_{h} & \text { outside } B_{r}\left(x_{h}\right), \\ \max \left\{\min \left\{u_{h}, M_{h}\right\}, m_{h}\right\} & \text { inside } B_{r}\left(x_{h}\right) .\end{cases}
$$

Since $x_{h} \in \Gamma_{h} \backslash \Gamma_{h}^{*}$, and in view (A.9) and of (A.16), we deduce that

$$
\left|\left\{u_{h} \neq \tilde{u}_{h}\right\} \backslash E^{h}\right|>0
$$

so that

$$
\int_{\Omega} f\left(x, \nabla \tilde{u}_{h}\right) \mathrm{d} x<\int_{\Omega} f\left(x, \nabla u_{h}\right) \mathrm{d} x,
$$

which is against the minimality of $u_{h}$. Then (A.17) holds.

Let $\partial_{\vartheta} u_{h}$ denote the angular derivative of $u_{h}$, that is, $\partial_{\vartheta} u_{h}:=\frac{\mathrm{d}}{\mathrm{d} \vartheta} u_{h}\left(x_{h}^{1}+\right.$ $\left.r \cos \vartheta, x_{h}^{2}+r \sin \vartheta\right)$. Setting $C_{h}:=\left\{s \in[0, r]: \mathcal{H}^{1}\left(\partial B_{s}\left(x_{h}\right) \cap \Gamma_{h}\right)=0\right\}$, by (A.17) we have for every $r \in C_{h}$

$$
\int_{0}^{2 \pi}\left|\partial_{\vartheta} u_{h}\right|^{p} \mathrm{~d} \vartheta \geqq 2 \pi \delta^{p} .
$$

Notice that $\left|C_{h}\right| \geqq r-\mathcal{H}^{1}\left(\Gamma_{h}\right)$. In fact we can obtain $\mathcal{H}^{1}\left(\Gamma_{h}\right)$ considering coverings of $\Gamma_{h}$ made up by disks, and taking the sum of the length of their boundaries: through suitable rotations we can move the disks in such a way that their centers lie on a fixed radius of $B\left(x_{h}, r\right)$, so that the relation follows. We deduce that

$$
\begin{aligned}
\int_{B_{r}\left(x_{h}\right)}\left|\nabla u_{h}\right|^{p} \mathrm{~d} x & \geqq \int_{0}^{r} \int_{0}^{2 \pi} s^{1-p}\left|\partial_{\vartheta} u_{h}\right|^{p} \mathrm{~d} \vartheta \mathrm{d} s \\
& \geqq \int_{C_{h}} \int_{0}^{2 \pi} s^{1-p}\left|\partial_{\vartheta} u_{h}\right|^{p} \mathrm{~d} \vartheta \mathrm{d} s \geqq 2 \pi \delta^{p} \int_{C_{h}} s^{1-p} \mathrm{~d} s \\
& \geqq 2 \pi \delta^{p} \int_{\mathcal{H}^{1}\left(\Gamma_{h}\right)}^{r} s^{1-p} \mathrm{~d} s
\end{aligned}
$$


Let us distinguish two cases, namely $p \geqq 2$ and $1<p<2$. If $p \geqq 2$, choosing $r=2 \mathcal{H}^{1}\left(\Gamma_{h}\right)$ we obtain

$$
\liminf _{h \rightarrow+\infty} \int_{B_{r_{h}}\left(x_{h}\right)} f\left(x, \nabla u_{h}\right) \mathrm{d} x \geqq 2 \pi \delta^{p} \ln 2 .
$$

But this is against (A.13): in fact for all $r$ such that $B_{r}(\bar{x}) \subseteq \Omega$ by (A.13) we have

$$
\begin{aligned}
\int_{B_{r}(\bar{x})} f(x, \nabla u) \mathrm{d} x & =\lim _{h \rightarrow+\infty} \int_{B_{r}(\bar{x})} f\left(x, \nabla u_{h}\right) \mathrm{d} x \\
& \geqq \lim _{h \rightarrow+\infty} \int_{B_{r_{h}}\left(x_{h}\right)} f\left(x, \nabla u_{h}\right) \mathrm{d} x \geqq 2 \pi \delta^{p} \ln 2,
\end{aligned}
$$

and this gives a contradiction for $r$ small enough. If $1<p<2$, then we have

$\liminf _{h \rightarrow+\infty} \int_{B_{r}\left(x_{h}\right)} f\left(x, \nabla u_{h}\right) \mathrm{d} x \geqq \liminf _{h \rightarrow+\infty} \frac{2 \pi \delta^{p}}{2-p}\left(r^{2-p}-\mathcal{H}^{1}\left(\Gamma_{h}\right)^{2-p}\right)=\frac{2 \pi \delta^{p}}{2-p} r^{2-p}$

from which by (A.13) we deduce that

$$
\int_{B_{r}(\bar{x})} f(x, \nabla u) \mathrm{d} x \geqq C r^{2-p}
$$

for some $C>0$. Since $\nabla u \in C^{0}\left(\bar{\Omega} ; \mathbb{R}^{2}\right)$ and $f$ satisfies (2.3) we get that

$$
\int_{B_{r}(\bar{x})} f(x, \nabla u) \mathrm{d} x \leqq \tilde{C} r^{2}
$$

for some $\tilde{C}>0$, which together with (A.19) gives a contradiction.

The case in which $\bar{x} \in \partial \Omega$ can be treated almost in the same way as the case $\bar{x} \in \Omega$. In fact it is sufficient to choose $r$ so small that

$$
\max _{B_{r}\left(x_{h}\right) \cap \partial_{D} \Omega} \psi-\min _{B_{r}\left(x_{h}\right) \cap \partial_{D} \Omega} \psi<\delta,
$$

and to take into account the fact that there exists a constant $C$ depending only on $\Omega$ such that

$$
\mathcal{H}^{1}\left(\partial B_{r_{h}}\left(x_{h}\right) \cap \Omega\right) \geqq C r_{h} .
$$

In this way, integrations involved in (A.18) can be performed on a set of angle $\vartheta$ which has a positive measure uniformly bounded from below, and the contradiction follows by the same arguments used above. 


\section{Appendix B: Uniform Poincaré and Poincaré-Korn inequalities}

In this section, we show that very basic arguments lead to the uniform Poincaré and Poincaré-Korn inequalities that are needed, respectively, in Sections 3 and 4.

To simplify, we will only consider the case of $N$-dimensional domains of the form

$$
Q_{f}=\left\{x=\left(x^{\prime}, x_{N}\right) \in \mathbb{R}^{N}: 0 \leqq x_{i} \leqq 1, i=1, \ldots, N-1,0 \leqq x_{N} \leqq f\left(x^{\prime}\right)\right\}
$$

where $f: Q^{\prime} \rightarrow[1, M]$ is an $L$-Lipschitz function. Here $Q^{\prime}$ is the $(N-1)$-dimensional cube $(0,1)^{N-1}$ and $L>0$ and $M>1$ are fixed constants. The adaptation of the arguments that we will present here to the "real" cases that are useful in the paper is straightforward. Also, for simplicity, we consider here the "linear" case $p=2$. However, the proofs would be identical with any other exponent $p \in(1,+\infty)$.

With a slight abuse in the notation we also identify $Q^{\prime}$ with the base of $Q_{f}$, that is, the subset $(0,1)^{N-1} \times\{0\}$ of $\partial Q_{f}$. We show that the following result holds:

Proposition 4. There exists a constant $C>0$ depending only on $L$ and $M$ such that

(i) For any $u \in H^{1}\left(Q_{f}\right)$ with $u=0$ on $Q^{\prime},\|u\|_{L^{2}\left(Q_{f}\right)} \leqq C\|\nabla u\|_{L^{2}\left(Q_{f}\right)}$;

(ii) For any $u \in H^{1}\left(Q_{f}\right)$ with $\int_{Q_{f}} u(x) \mathrm{d} x=0,\|u\|_{L^{2}\left(Q_{f}\right)} \leqq C\|\nabla u\|_{L^{2}\left(Q_{f}\right)}$;

(iii) For any $\mathbf{u} \in H^{1}\left(Q_{f} ; \mathbb{R}^{N}\right)$ with $\mathbf{u}=0$ on $Q^{\prime},\|\mathbf{u}\|_{L^{2}\left(Q_{f}\right)} \leqq C\|e(\mathbf{u})\|_{L^{2}\left(Q_{f}\right)}$;

(iv) For any $\mathbf{u} \in H^{1}\left(Q_{f} ; \mathbb{R}^{N}\right)$ with both $\int_{Q_{f}} \mathbf{u}(x) \mathrm{d} x=0$ and $\int_{Q_{f}} x \times \mathbf{u}(x) \mathrm{d} x=$ 0 , one has $\|\mathbf{u}\|_{L^{2}\left(Q_{f}\right)} \leqq C\|e(\mathbf{u})\|_{L^{2}\left(Q_{f}\right)}$.

In the last assertion, $x \times \mathbf{u}$ is the skew-symmetric matrix $\left(x_{i} \mathbf{u}_{j}-x_{j} \mathbf{u}_{i}\right)_{i, j=1}^{N}$, and the condition means that $\mathbf{u}$ is orthogonal (in $L^{2}$ ) to the rigid motions (of the form a $+B x$ with $B$ skew-symmetric).

Let us sketch the proof of this proposition. First of all, the proof of point (i) is standard (by integration along vertical lines starting from $Q^{\prime}$ ) and it is well known that the constant $C$, in this case, only depends on $M$ ( $f$ could then be any l.s.c. function below $M$ ). In the same way, the proof of (iii) is significantly simpler than the proof of (iv) (note however that it does require that $f$ is Lipschitz and $C$ will depend on both $M$ and $L$ ), and we will not discuss it. (See [7] for a detailed proof, in dimension two).

To prove (ii) one first establishes the following inequality: there exists $C_{0}$ depending only on $M$ such that for any $f$ and any $u \in H^{1}\left(Q_{f}\right)$, one has

$$
\int_{Q_{f}} u(x)^{2} \mathrm{~d} x \leqq C_{0}\left(\int_{A} u(x)^{2} \mathrm{~d} x+\int_{Q_{f}}|\nabla u(x)|^{2} \mathrm{~d} x\right),
$$

where $A$ denotes the set $Q^{\prime} \times(0,1)$ (the important fact here being that $A$ is an open set that belongs to all the domains $Q_{f}$, for all admissible $f$ ). The proof of (B.1), again, is standard. It relies on integration along vertical lines starting from the base $Q^{\prime}$ and on the obvious fact that for any $b \in[1, M]$ and any $v \in C^{1}(0, b)$,

$$
\int_{0}^{y} v(t)^{2} \mathrm{~d} t \leqq 2 M \int_{0}^{1} v(t)^{2} \mathrm{~d} t+2 M^{2} \int_{0}^{b} v^{\prime}(t)^{2} \mathrm{~d} t .
$$


Now, if (ii) is not true, it means that there exists functions $f_{n}$ and $u_{n}$ with $1 \leqq f_{n} \leqq M, f_{n} L$-Lipschitz, $u_{n} \in H^{1}\left(Q_{f_{n}}\right), \int_{Q_{f_{n}}} u_{n} \mathrm{~d} x=0$, and

$$
\left\|u_{n}\right\|_{L^{2}\left(Q_{f_{n}}\right)} \geqq n\left\|\nabla u_{n}\right\|_{L^{2}\left(Q_{f_{n}}\right)}
$$

for any $n$.

Without loss of generality we may renormalize $u_{n}$ so that $\int_{A} u_{n}^{2} \mathrm{~d} x=1$. Then, by (B.1) we find

$$
\left\|\nabla u_{n}\right\|_{L^{2}\left(Q_{f_{n}}\right)} \leqq \frac{1}{n}\left\|u_{n}\right\|_{L^{2}\left(Q_{f_{n}}\right)} \leqq \frac{\sqrt{C_{0}}}{n}\left(1+\left\|\nabla u_{n}\right\|_{L^{2}\left(Q_{f_{n}}\right)}\right) .
$$

If we extend both $u_{n}$ and $\nabla u_{n}$ with the value 0 outside of $Q_{f_{n}}$, this inequality shows that $\nabla u_{n}$ goes to zero strongly in $L^{2}\left(Q_{M} ; \mathbb{R}^{N}\right)\left[Q_{M}=Q^{\prime} \times(0, M)\right]$, and, up to a subsequence, that there exists $u \in L^{2}\left(Q_{M}\right)$ such that $u_{n} \rightarrow u$ weakly in $L^{2}\left(Q_{M}\right)$.

On the other hand, by Rellich's theorem, $u_{n} \rightarrow u$ strongly in $L^{2}(A)$ and since $\nabla u=0$ in $A$ and $\int_{A} u^{2}=1, u$ is the constant $\pm 1 / \sqrt{|A|}$.

Now, since the functions $f_{n}$ are uniformly equibounded and equicontinuous, up to a further subsequence, we may assume also that $f_{n}$ converges to some $f$ uniformly. It is now easy to check that $u \in H^{1}\left(Q_{f}\right), u=0$ outside of $Q_{f}$, and $\nabla u=0$ (the limit of $\nabla u_{n}$ ) in $Q_{f}$ so that $u$ is a constant in $Q_{f}$. We deduce that $u=(1 / \sqrt{|A|}) \chi_{Q_{f}}$. Now, for each $n$, one had $\int_{Q_{f_{n}}} u_{n} \mathrm{~d} x=\int_{Q_{M}} u_{n} \mathrm{~d} x=0$, hence in the limit $\int_{Q_{M}} u \mathrm{~d} x=0 \neq\left|Q_{f}\right| / \sqrt{|A|}$, a contradiction. Hence (ii) must be true.

We observe here that (ii) holds in fact as long as $f$ belongs to a fixed set of functions which is compact in $C^{0}\left(Q^{\prime},[1,+\infty)\right.$ ) (the constant $C$ depending only on this compact set). The case of $L$-Lipschitz functions uniformly bounded by the constant $M$ is a particular case. On the other hand, for the Poincaré-Korn inequalities (iii) and (iv), the fact that the functions $f$ are uniformly Lipschitz seems to be essential, as we now show.

Let us now prove (iv). It is enough to show that the vectorial version of (B.1) holds, that is,

$$
\int_{Q_{f}}|\mathbf{u}(x)|^{2} \mathrm{~d} x \leqq C_{0}\left(\int_{A}|\mathbf{u}(x)|^{2} \mathrm{~d} x+\int_{Q_{f}}|e(\mathbf{u})(x)|^{2} \mathrm{~d} x\right) .
$$

This will be shown, again, by integration along lines and using (B.2), however, this time, it is not sufficient to consider only vertical lines starting from $Q^{\prime}$. Indeed, one has for any smooth vectorial field $\mathbf{u} \in C^{1}\left(Q_{f}\right)$ that

$$
\mathrm{d}(\mathbf{u}(x+s \xi) \cdot \xi) / \mathrm{d} s=(e(\mathbf{u})(x+s \xi) \xi) \cdot \xi
$$

for any $x \in Q_{f}, \xi \in \mathbb{S}^{N-1}$, and $s \in \mathbb{R}$ such that $x+s \xi \in Q_{f}$. Hence, integration along vertical lines will control the component $u_{N}$ of $\mathbf{u}=\left(u_{1}, \ldots, u_{N}\right)$. To control the other components, one needs to integrate along lines in at least $N-1$ other independent directions (as is done in [7]).

Given $p>\max \{L, 2 M\}$ let us consider, for any $i=1, \ldots, N-1$, the vectors

$$
\xi_{i}^{ \pm}=\frac{1}{\sqrt{1+p^{2}}}(0, \ldots, 0, \pm 1,0, \ldots, p)
$$


where \pm 1 appears at the $i$ th position. Given $i \leqq N-1$, if we considers the lines starting from $Q^{\prime}$ in the direction $\xi_{i}^{+}$, we see that they "see" all points $x \in Q_{f}$ with $x_{i} \geqq 1 / 2$. On the other hand, the lines starting from $Q^{\prime}$ in the direction $\xi_{i}^{-}$ "see" all the points with $x_{i} \leqq 1 / 2$. Integrating along these lines and using (B.2), one controls the $L^{2}$-norms on one half of the domain $Q_{f}$ of $\left(u_{i}+p u_{N}\right) / \sqrt{1+p^{2}}$ and, on the other half, of $\left(-u_{i}+p u_{N}\right) / \sqrt{1+p^{2}}$. Together with the control of $\int_{Q_{f}} u_{N}^{2} \mathrm{~d} x$ obtained previously, this shows that one can control $\int_{Q_{f}} u_{i}^{2} \mathrm{~d} x$ with the right-hand side of (B.3). Repeating this argument for all $i$, we find that (B.3) holds, with now a constant that depends on $M$ and $L$, through $p$.

We deduce, exactly as before, that (iv) holds.

Acknowledgements. The authors wish to thank Gilles Francfort, Jean-JacQues Marigo, and Vincenzo Nesi for interesting discussions. The first and third authors are partially funded by the MULTIMAT European network MRTN-CT_2004-505226.

\section{References}

1. Ambrosio, L.: A compactness theorem for a new class of functions of bounded variations. Boll. Un. Mat. Ital. 3-B, 857-881 (1989)

2. Ambrosio, L.: Existence theory for a new class of variational problems. Arch. Ration. Mech. Anal. 111, 291-322 (1990)

3. Ambrosio, L.: A new proof of the SBV compactness theorem. Calc. Var. Partial Differ. Equ. 3, 127-137 (1995)

4. Ambrosio, L., Fusco, N., Pallara, D.: Functions of Bounded Variations and Free Discontinuity Problems. Clarendon, Oxford, 2000

5. Ambrosio, L., Caselles, V., Masnou, S., Morel, J.-M.: Connected components of sets of finite perimeter and applications to image processing. J. Eur. Math. Soc. (JEMS) 3, 39-92 (2001)

6. Astala, K.: Area distortion of quasiconformal mappings. Acta Math. 103(1), 37-60 (1994)

7. Bonnetier, E., Chambolle, A.: Computing the equilibrium configuration of epitaxially strained crystalline films. SIAM J. Appl. Math. 62, 1093-1121 (2002) (electronic)

8. Boyarski, B.: Homeomorphic solutions of Beltrami systems (Russian). Dokl. Akad. Nauk SSSR (N.S.) 102, 661-664 (1955)

9. BoyARSKI, B.: Generalized solutions of a system of differential equations of first order and of elliptic type with discontinuous coefficients (Russian). Mat. Sb. N.S. 43(85), 451-503 (1957)

10. BRezis, H.: Opérateurs maximaux monotones et semi-groupes de contractions dans les espaces de Hilbert. North-Holland, Amsterdam, 1973

11. Caffarelli, L., Peral, I.: On $W^{1, p}$ estimates for elliptic equations in divergence form. Commun. Pure Appl. Math. 51, 1-21 (1998)

12. Сhambolle, A.: A density result in two-dimensional linearized elasticity, and applications. Arch. Ration. Mech. Anal. 167, 211-233 (2003)

13. Dal Maso, G., Morel, J.-M., Solimini, S.: A variational method in image segmentation: existence and approximation results. Acta Math. 168(1-2), 89-151 (1992)

14. Dal Maso, G., Toader, R.: A model for the quasistatic growth of brittle fractures: existence and approximation results. Arch. Ration. Mech. Anal. 162, 101-135 (2002)

15. Dal Maso, G., Toader, R.: A model for the quasi-static growth of brittle fractures based on local minimization. Math. Models Methods Appl. Sci. 12, 1773-1799 (2002) 
16. David, G.: Singular Sets of Minimizers for the Mumford-Shah Functional. Progress in Mathematics, Birkhaüser, Basel, 2005

17. De Giorgi, E., Carriero, M., Leaci, A.: Existence theorem for a minimum problem with free discontinuity set. Arch Ration. Mech. Anal. 108, 195-218 (1989)

18. Eвobisse, F., Ponsiglione, M.: A duality approach for variational problems in domains with cracks. J. Convex Anal. 11, 17-40 (2004)

19. Francfort, G.A., LARSEn, C.J.: Existence and convergence for quasistatic evolution in brittle fracture. Commun. Pure Appl. Math. 56, 1465-1500 (2003)

20. FrancFort, G.A., Marigo, J.-J.: Revisiting brittle fractures as an energy minimization problem. J. Mech. Phys. Solids 46, 1319-1342 (1998)

21. Grisvard, P.: Elliptic Problems in Nonsmooth Domains. Pitman, Boston, 1985

22. LeOnetti, F., Nesi, V.: Quasiconformal solutions to certain first order systems and the proof of a conjecture of G. W. Milton. J. Math. Pure Appl. 76, 109-124 (1997)

23. Li, Y., NiRENBERG, L.: Estimates for elliptic systems from composite material. Dedicated to the memory of Jürgen K. Moser. Commun. Pure Appl. Math. 56, 892-925 (2003)

24. LI, Y., Vogelius, M.: Gradient estimates for solutions to divergence form elliptic equations with discontinuous coefficients. Arch. Ration. Mech. Anal. 153, 91-151 (2000)

25. Maddalena, F., Solimini, S.: Lower semicontinuity properties of functionals with free discontinuities, Arch. Ration. Mech. Anal 159(4), 273-294 (2001)

26. Meyers, N.G.: An $L^{p}$-estimate for the gradient of solutions of second order elliptic divergence equations. Ann. Scuola Norm. Sup. Pisa 17, 189-206 (1963)

27. Mielke, A.: Analysis of energetic models for rate-independent materials. In: Proceedings of the International Congress of Mathematicians, Vol. III (Beijing, 2002), pp. 817-828, Higher Ed. Press, Beijing, 2002

28. Morel, J.-M., Solimini, S.: Variational Methods in Image Segmentation. Birkhaüser, Boston, 1995

29. Mumford, D., Shah, J.: Optimal approximations by piecewise smooth functions and associated variational problems. Commun. Pure Appl. Math. 42, 577-685 (1989)

30. Rockafellar, R.T.: Convex Analysis. Princeton Mathematical Series, No. 28 Princeton University Press, Princeton, 1970

31. Solimini, S.: Simplified excision techniques for free discontinuity problems in several variables. J. Funct. Anal. 151, 1-34 (1997)

CMAP, Ecole Polytechnique, CNRS, 91128 Palaiseau, France.

e-mail: antonin.chambolle@ polytechnique.fr

and

Dipartimento di Matematica, Facoltà di Ingegneria,

Università degli Studi di Brescia,

Via Valotti 9, 25133 Brescia, Italy. e-mail: alessandro.giacomini@ing.unibs.it

and

Max Planck Institute for Mathematics in the Sciences, Inselstrasse 22, 04103 Leipzig, Germany. e-mail: marcello.ponsiglione@ @mis.mpg.de

(Received November 25, 2005 / Accepted October 20, 2006)

Published online November 13, 2007 - (C) Springer-Verlag (2007) 